\title{
Solution Structure and Ultrafast Vibrational Relaxation of the PtPOP Complex Revealed by SCF-QM/MM Direct Dynamics Simulations
}

Levi, Gianluca; Pápai, Mátyás Imre; Henriksen, Niels E.; Dohn, Asmus O.; Møller, Klaus B.

Published in:

The Journal of Physical Chemistry Part C

Link to article, DOI:

10.1021/acs.jpcc.8b00301

Publication date:

2018

Document Version

Peer reviewed version

Link back to DTU Orbit

Citation (APA):

Levi, G., Pápai, M. I., Henriksen, N. E., Dohn, A. O., \& Møller, K. B. (2018). Solution Structure and Ultrafast Vibrational Relaxation of the PtPOP Complex Revealed by SCF-QM/MM Direct Dynamics Simulations. The Journal of Physical Chemistry Part C, 122(13), 7100-7119. https://doi.org/10.1021/acs.jpcc.8b00301

\section{General rights}

Copyright and moral rights for the publications made accessible in the public portal are retained by the authors and/or other copyright owners and it is a condition of accessing publications that users recognise and abide by the legal requirements associated with these rights.

- Users may download and print one copy of any publication from the public portal for the purpose of private study or research.

- You may not further distribute the material or use it for any profit-making activity or commercial gain

- You may freely distribute the URL identifying the publication in the public portal 


\section{Solution Structure and Ultrafast Vibrational}

\section{Relaxation of the PtPOP Complex Revealed by $\triangle$ SCF-QM/MM Direct Dynamics Simulations}

Gianluca Levi, ${ }^{\dagger}$ Mátyás Pápai, ${ }^{\dagger,}$ Niels E. Henriksen, ${ }^{\dagger}$ Asmus O. Dohn, ${ }^{\dagger}{ }^{\top}$ and Klaus B. Møller*,†

$\dagger$ Department of Chemistry, Technical University of Denmark, Kemitorvet 206, 2800 Kongens Lyngby, Denmark

$\ddagger$ Wigner Research Centre for Physics, Hungarian Academy of Sciences, P.O. Box 49, H-1525 Budapest, Hungary

ICurrent address: Faculty of Physical Sciences, University of Iceland, 107 Reykjavik, Iceland

E-mail: kbmo@kemi.dtu.dk 


\begin{abstract}
Recent ultrafast experiments have unveiled the time scales of vibrational cooling and decoherence upon photoexcitation of the diplatinum complex $\left[\mathrm{Pt}_{2}\left(\mathrm{P}_{2} \mathrm{O}_{5} \mathrm{H}_{2}\right)_{4}\right]^{4-}$ (PtPOP) in solvent. Here we contribute to the understanding of the structure and dynamics of the lowest-lying singlet excited state of the model photocatalyst by performing potential energy surface (PES) calculations and Born-Oppenheimer Molecular Dynamics (BOMD) simulations in gas phase and water. Solvent effects were treated using a multiscale quantum mechanics/molecular mechanics (QM/MM) approach. Fast sampling was achieved with a modified version of $\Delta \mathrm{SCF}$ implemented in the Gridbased Projector Augmented Wave (GPAW) density functional theory (DFT) code. The known structural parameters and the PESs of the first singlet and triplet excited states are correctly reproduced. Besides, the simulations deliver clear evidence that pseudorotation of the ligands in the excited state leads to symmetry lowering of the $\mathrm{Pt}_{2} \mathrm{P}_{8}$ core. Coherence decay of Pt-Pt stretching vibrations in solution was found to be governed by vibrational cooling, in agreement with previous ultrafast experiments. We also show that the flow of excess Pt-Pt vibrational energy is first directed towards vibrational modes involving the ligands, with the solvent favouring intramolecular vibrational energy redistribution (IVR). The results are supported by thorough vibrational analysis in terms of generalized normal modes.
\end{abstract}

\title{
Introduction
}

The field of photocatalysis has witnessed increased interest in transition metal complexes over the last years. Indeed, stability in solution and remarkable photophysical properties make many of them attractive candidates for application in solar harvesting devices. ${ }^{1-5}$ The continuous demand for more efficient and tunable photocatalytic systems has stimulated a vast amount of experimental studies on solvated metal complexes ${ }^{6-8}$. Taking full advantage of their photocatalytic properties requires, in particular, an understanding of the structurefunction relationships and mechanisms behind ultrafast light-induced reactions in complex 
environments. Photo-induced molecular processes can now be observed at an unprecedented level of detail, thanks to recent advances in time-resolved techniques. Among them, improvements to the time resolution of ultrafast optical and X-ray spectroscopies and X-ray diffraction measurements performed at X-ray Free Electron Laser (XFEL) facilities down to the femtosecond regime have led to experiments able to follow the evolution of vibrational wave packets or the solvation dynamics in photoexcited prototypical metal complexes in real-time. ${ }^{9-16}$ However, these novel experiments cover grounds often dominated by complex interplays between vibrational relaxation, solvent effects and electronic couplings, which are not known a priori. Therefore, linking experimental observations to mechanistic frameworks can only be accomplished with the help of solid theoretical and modelling strategies. Moreover, even when the interpretation of an experiment is facilitated by prior photophysical knowledge or by employing simple phenomenological models, a variety of complementary techniques are needed to assemble a complete atomistic and energetic picture of the early stages of the investigated dynamics. In this context, advanced computational methods capable of connecting multiple time-resolved observables, while delivering new mechanistic insights into the underlying physical processes, play an important role in complementing ultrafast experiments.

One of the main challenges associated with the a priori determination of the mechanisms of the ultrafast excited-state dynamics of complex molecular systems is represented by the time scales one is able to simulate while retaining accuracy. Broadly speaking, much of the efforts of the theoretical community to address this problem have been directed towards the development and application of two computational frameworks of choice: methods that solve the time-dependent Schrödinger equation for the nuclei using precomputed potential energy surfaces (PESs), ${ }^{17-23}$ and methods based on classical propagation of the nuclei with on-thefly evaluation of energies and forces at $a b$ initio level. ${ }^{24-33}$ Quantum dynamics approaches have proven useful in deciphering some aspects of the excited-state decay pathways of photocatalytic metal complexes, particularly concerning non-adiabatic electronic transitions. ${ }^{17,20}$ 
However, the outcome of this kind of simulation relies on the selection of a small number of vibrational modes along which the dynamics is restricted. Furthermore, solvent effects in quantum wave packet simulations are usually accounted for in an implicit manner, ${ }^{19,21}$ thus neglecting any explicit solvation dynamics effect.

In the present work, we have chosen an approach following the second route: ab initio classical dynamics. This strategy allows, in principle, to efficiently explore the full, unconstrained space of nuclear configurations and to include explicit solvent effects in a multiscale fashion. Recently we have presented ${ }^{34}$ a density functional theory (DFT) implementation of on-the-fly QM/MM Born-Oppenheimer Molecular Dynamics (BOMD) that is capable of extensive sampling of equilibrium thermal properties and out-of-equilibrium dynamics of solvated transition metal complexes. ${ }^{12,29,34,35}$ The implementation is available within the Atomic Simulation Environment (ASE) ${ }^{36,37}$ and uses the computationally efficient Gridbased Projector Augmented Wave (GPAW) $\operatorname{code}^{38,39}$ for the DFT part. It has been already successfully applied to study the ultrafast internal vibrational dynamics and to obtain a picture of solvent-driven electronic dynamics in bimetallic photoactive complexes, ${ }^{29,35}$ and has proven decisive in establishing a robust interpretation of the solvation dynamics at catalytic sites observed in ultrafast diffuse X-ray scattering (XDS) data. ${ }^{12}$ For all cases, the excited states of interest were described using the spin unrestricted DFT formalism. In some of the investigated systems, the observed ultrafast dynamics following photoexcitation was known to take place on an excited state of the same spin multiplicity as the ground state, usually a singlet. This implied that the simulations had to approximate the dynamics by propagating the system on the lowest excited state of a different spin multiplicity by assuming parallel potential energy surfaces along the dominant vibrational motions. However, even in systems for which the latter assumption was demonstrated to be valid, the dynamics in the two states can still be different if their energies are such that they lie in regions of different density of states, as recently shown by Monni et al. ${ }^{10}$ These authors compared the coherence decay of vibrational wave packets in the first singlet and triplet excited states 
of diplatinum complexes in solution observed in ultrafast optical measurements, and found significant differences despite parallel potential energy surfaces.

The need to be able to reliably compare simulations to experimental results calls for an extension of the QM/MM BOMD method in ASE/GPAW to encompass states of arbitrary spin multiplicity. With this perspective in mind, we have extended the capabilities of the code by coupling it to a single-determinant DFT description of the excited states based on the $\triangle \mathrm{SCF}$ approach, ${ }^{40}$ which carries no extra computational cost with respect to ground-state DFT. $\triangle \mathrm{SCF}$ is gaining increasing popularity in the study of the excited states of both organic chromophores ${ }^{41-44}$ and transition metal complexes. ${ }^{45,46}$ This renewed interest is motivated in part by the growing demand for computationally cheap strategies for simulating with sufficient accuracy the excited-state structure and dynamics of large systems, for which high-level multireference methods are not yet a viable choice. The reliability of $\triangle \mathrm{SCF}$ as applied to study the structure and dynamics of small molecules, organic dyes and even biological systems, has been assessed with respect to vibrational analysis, ${ }^{47}$ exploration of PESs, ${ }^{44,48}$ as well as dynamics in solution within QM/MM MD frameworks. ${ }^{42,49}$ On the other hand, to our knowledge, no studies exist that investigate the ability of the method to predict the structural dynamics of transition metal complexes, even though the performances of $\triangle \mathrm{SCF}$ for excitation energies and simulations of UV-vis spectra of metal-containing molecular systems are not inferior to those achieved when applied to organic molecules. ${ }^{45,46}$

In a previous computational work ${ }^{34}$ we applied the QM/MM BOMD scheme in ASE/GPAW to the ground-state structural dynamics of the diplatinum(II) complex $\left[\mathrm{Pt}_{2}\left(\mathrm{P}_{2} \mathrm{O}_{5} \mathrm{H}_{2}\right)_{4}\right]^{4-}$, abbreviated PtPOP, in aqueous solution. In the study we collected almost half a nanosecond of equilibrated BOMD data, which permitted a statistically unbiased comparison of thermally averaged structural and dynamical properties with available experimental results, showing remarkable agreement. Moreover, we characterized in detail the solvent shell structure around the complex, which had been previously debated due to the lack of direct experimental evidence and statistically relevant computational insights. Here we investigate 
PtPOP in water in the first singlet excited state using our new $\triangle$ SCF-QM/MM BOMD implementation.

Owing to its nuclear and electronic structures, PtPOP is the prototype system of choice for photophysical studies within a family of highly photoreactive $\mathrm{d}^{8}-\mathrm{d}^{8}$ binuclear complexes. ${ }^{7}$ The UV-vis absorption spectrum of PtPOP in crystal and different solvents exhibits an intense band around $370 \mathrm{~nm}$ and a weaker band around $450 \mathrm{~nm}$ that are attributed to electronic transition from the $\mathrm{HOMO} \mathrm{d} \sigma^{*}$ antibonding to the LUMO $\mathrm{p} \sigma$ bonding metal-metal orbital. ${ }^{50-52}$ As a result of the nature of the excitation, the first singlet and triplet excited states $\left(\mathrm{S}_{1}\right.$ and $\mathrm{T}_{1}$ ), having $\mathrm{d} \sigma^{*} \rightarrow \mathrm{p} \sigma$ character, feature a significantly shortened Pt-Pt distance. Reported experimental values for the contraction in crystal and solution lie in the range $0.19-0.31 \AA .{ }^{51,53-57}$ It is well established, based on the vibronic progression of lowtemperature UV-vis $\mathrm{S}_{0} \rightarrow \mathrm{S}_{1}$ and $\mathrm{S}_{0} \rightarrow \mathrm{T}_{1}$ absorption bands, ${ }^{50}$ that the potential energy surfaces of $\mathrm{S}_{1}$ and $\mathrm{T}_{1}$ along the Pt-Pt coordinate are parallel. Moreover, these states are found, from experiments ${ }^{50,51}$ and previous DFT studies, ${ }^{58,59}$ to be separated by a relatively large energy gap of around $0.65 \mathrm{eV}$, and isolated from other electronic states. The electronic structure of the complex, together with the fact that direct spin-orbit coupling (SOC) between $S_{1}$ and $T_{1}$ is forbidden for symmetry reasons, ${ }^{58,60}$ accounts for intersystem crossing (ISC) times between 11.0 and 101.5 ps ${ }^{14,50,61}$ depending on solvent and temperature. Besides, the lifetime of $T_{1}$ is found to be on the order of microseconds. ${ }^{50}$ Ultimately, it is this state that has catalytic activity, being able to abstract hydrogen and halogen atoms from different substrates. ${ }^{7,62}$

The peculiar photophysical properties of PtPOP have been exploited to characterize, by femtosecond optical measurements, the evolution of wave packet coherent vibrations along the Pt-Pt coordinate in $\mathrm{S}_{1}{ }^{14}$ and recently also in the $\mathrm{T}_{1}$ state. ${ }^{10}$ Some of the aspects of the ultrafast relaxation following excitation in the $\mathrm{S}_{1}$ state in different solvents where uncovered in a combined fluorescence up-conversion and broadband transient absorption (TA) study by van der Veen et al. ${ }^{14}$ It was found that the coherence decay of vibrational wave packets with 
a period of $\sim 224$ fs takes place concomitantly with vibrational cooling over a remarkably long time of 1-2 ps. The observations were interpreted as a signature of the strong harmonicity of the potential along the Pt-Pt coordinate, which in turn is due to the rigidity of the cage of P-O-P ligands, and effective shielding from random solvent fluctuations provided by the latter. Despite the fact that the experiments could characterize the time scales of vibrational coherence, cooling and ISC in solution, the mechanistic details behind these processes are far from being well understood. Hypotheses of mechanisms of vibrational cooling have been put forward, but they are not based on direct experimental evidence; rather they rely on the observation of solvent trends ${ }^{14}$ or the comparison with the behaviour of diplatinum systems with modified ligands under similar experimental conditions. ${ }^{10}$ Thus, van der Veen et al. ${ }^{14}$ assign differences in the vibrational decay rates for excitation in the $\mathrm{S}_{1}$ state observed for different solvents to direct solute-solvent interactions that can only occur along the open axial Pt-Pt coordination sites of the molecule. More recently, Monni et al. ${ }^{10}$ seem to exclude this possibility. These authors argue that, since no big differences with respect to the decoherence times of a perfluoroborated derivative of PtPOP for which the bulkier ligands offer better shielding of the Pt atoms from the environment were observed, the origin of coherence decay must arise from anharmonic couplings of the Pt-Pt motion with other internal vibrational modes. The mechanism of ISC from $\mathrm{S}_{1}$ to $\mathrm{T}_{1}$ in PtPOP is also a recurrent subject of discussion in the PtPOP literature. ${ }^{7,14,58,60}$ All recent experimental indications seem to point in the direction of a possible involvement of a dark mode that would lower the $D_{4 h}$ symmetry of the $\mathrm{Pt}_{2} \mathrm{P}_{8}$ core of the complex, allowing for direct SOC or lowering the energy of other triplet states, but this mode has never been observed experimentally. The scenario is complicated by the fact that up to now no experimental method has been able to reliably assess the changes affecting the structure of the ligands or the presence of large amplitude distortions in the excited state in solution.

As experimental techniques with atomistic resolution start putting hitherto unexplored sub-picosecond intramolecular structural and solvation processes under the microscope, de- 
veloping efficient computational methods capable of providing insights into the underlying physical mechanisms becomes of utmost importance. With this in mind, the primary objective of our work was to extend the QM/MM BOMD simulation framework to encompass states of arbitrary spin multiplicity using $\triangle \mathrm{SCF}$ implemented in GPAW. The current status of knowledge of the photophysics of PtPOP allows us to unambiguously assess the approach for statistically robust predictions of the structure and dynamics of the metal-centered excited state of $\mathrm{d}^{8}-\mathrm{d}^{8}$ transition metal complexes. At the same time, it leaves room for moving forward in the understanding of the microscopic mechanisms governing ultrafast relaxation in the $\mathrm{S}_{1}$ state of the complex. With respect to this, the present investigation was aimed at (i) elucidating structural distortions involving the ligands during the excited-state dynamics; (ii) characterizing the solvent shell response; (iii) assessing whether the ultrafast relaxation is exclusively governed by specific solute-solvent interactions or whether energy-accepting modes are also playing a role as mediators in the transfer of energy to the solvent. This was achieved by using $\triangle \mathrm{SCF}$ in extensive nonequilibrium gas-phase and solution-phase simulations in conjunction with a detailed vibrational analysis based on a decomposition of the time-dependent vibrational kinetic energy in generalized normal mode components. ${ }^{63}$ The solvent chosen for the investigation is water, for which accurate time-resolved excitedstate X-ray scattering ${ }^{54}$ and spectroscopic ${ }^{14}$ data are available and can be used to assess the validity of the simulations.

\section{Computational methods}

\section{$\triangle \mathrm{SCF}$ Implementation}

$\triangle \mathrm{SCF}$ excited states are represented by single Slater determinants that are constructed by constraining the orbital occupation numbers to non-ground-state configurations. ${ }^{40}$ Hence, when the approach is used within Kohn-Sham (KS) DFT, it consists in solving the variational KS equations for a set of KS orbitals $\psi_{k}(\mathbf{r})$ with constrained occupations. For standard 
$\triangle \mathrm{SCF}$ schemes based on promotion of a single electron from an occupied orbital $\psi_{a}(\mathbf{r})$ of the ground state to a virtual orbital $\psi_{b}(\mathbf{r})$, the electronic density of a system of $N_{e}$ electrons can be expressed as:

$$
\rho(\mathbf{r})=\sum_{i=1}^{N_{e}}\left(1-\delta_{a i}\right)\left|\psi_{i}(\mathbf{r})\right|^{2}+\sum_{j=N_{e}+1}^{n} \delta_{b j}\left|\psi_{j}(\mathbf{r})\right|^{2}
$$

where $\delta_{a i}$ and $\delta_{b j}$ are Kronecker deltas, and $n$ is the total number of orbitals included in the calculation. When the energy separation of $\psi_{a}(\mathbf{r})$ and $\psi_{b}(\mathbf{r})$ from other orbitals is sufficiently large, the KS equations with a density given by (1) will converge to the lowest electronic state of a given symmetry. However, variational convergence of the density can be difficult when state degeneracies are present. This can make $\triangle \mathrm{SCF}$ PES scans problematic for systems with high density of states, such as transition metal complexes. The issue of degeneracy in $\triangle \mathrm{SCF}$ calculations has been recently addressed by Maurer et al. ${ }^{44,64}$ with a modification of the ordinary $\triangle \mathrm{SCF}$ constraints to Gaussian smeared constraints affecting all orbitals that lie close in energy to the target orbitals $\psi_{a}(\mathbf{r})$ and $\psi_{b}(\mathbf{r})$. The method has been demonstrated to be able to deliver, when applied to azobenzene using generalized gradient approximation (GGA) functionals, ${ }^{44} \mathrm{PES}$ topologies close to conical intersections (CIs) of quality comparable to those obtained using higher level Coupled Cluster Singles and Doubles calculations. The proven robustness and flexibility of this $\triangle \mathrm{SCF}$ strategy are promising in view of its application in excited-state QM/MM BOMD simulations to ensure stable convergence of the density at each step of the dynamics. In order to explore this possibility, we have implemented $\triangle \mathrm{SCF}$ with Gaussian smeared constraints in a development branch of GPAW that can be used in conjunction with the new QM/MM module in ASE. ${ }^{34}$ At each step of an SCF cycle, the electronic density is computed from a modified form of equation (1):

$$
\rho(\mathbf{r})=\sum_{i=1}^{N_{e}}\left(1-g_{a}\left(\epsilon_{i}\right)\right)\left|\psi_{i}(\mathbf{r})\right|^{2}+\sum_{j=N_{e}+1}^{n} g_{b}\left(\epsilon_{j}\right)\left|\psi_{j}(\mathbf{r})\right|^{2}
$$


where $g_{a}\left(\epsilon_{i}\right)$ and $g_{b}\left(\epsilon_{j}\right)$ are Gaussian functions of the energies of the KS orbitals:

$$
\begin{aligned}
& g_{a}\left(\epsilon_{i}\right)=\frac{1}{N_{a}} \exp \left\{-\frac{\left(\epsilon_{i}-\epsilon_{a}\right)^{2}}{2 \sigma^{2}}\right\} \\
& g_{b}\left(\epsilon_{j}\right)=\frac{1}{N_{b}} \exp \left\{-\frac{\left(\epsilon_{j}-\epsilon_{b}\right)^{2}}{2 \sigma^{2}}\right\}
\end{aligned}
$$

The normalization factors for $g_{a}\left(\epsilon_{i}\right)$ and $g_{b}\left(\epsilon_{j}\right)$ are calculated by requiring that:

$$
\begin{gathered}
\sum_{i=1}^{N_{e}} g_{a}\left(\epsilon_{i}\right)=1 \\
\sum_{j=N_{e}+1}^{n} g_{b}\left(\epsilon_{j}\right)=1
\end{gathered}
$$

such that it satisfies conservation of the total number of electrons:

$$
\sum_{i=1}^{N_{e}}\left(1-g_{a}\left(\epsilon_{i}\right)\right)+\sum_{j=N_{e}+1}^{n} g_{b}\left(\epsilon_{j}\right)=N_{e}
$$

The parameter $\sigma$ controls the extent of the smearing, and can in principle be varied during the SCF cycle until satisfactory convergence is achieved. Employing the $\triangle \mathrm{SCF}$ implementation with the $\mathrm{QM} / \mathrm{MM}$ electrostatic embedding scheme presented in ${ }^{34}$ is straightforward. In fact, the electronic density of equation (2) can be directly used in the SCF cycle with the Hamiltonian including the external potential of the classical point charges of the solvent, and, once the converged quantities are obtained, all energy gradients needed to perform MD simulations can be computed using the routines available for ground-state DFT.

\section{Vibrational analysis from BOMD trajectories}

In order to attain a picture of intramolecular vibrational energy redistribution (IVR) during the $\triangle \mathrm{SCF}$ nonequilibrium vacuum and $\mathrm{QM} / \mathrm{MM}$ dynamics simulations, we performed a vibrational analysis of the trajectories according to the method proposed by Strachan. ${ }^{63,65}$ 
Following Strachan, ${ }^{63}$ generalized normal modes $Q_{i}$ defined as vibrational modes whose time evolution is uncorrelated to each other (and hence are not harmonic in general):

$$
\left\langle\dot{Q}_{i}(t) \dot{Q}_{j}(t)\right\rangle \propto \delta_{i j}
$$

where $\delta_{i j}$ is the Kronecker delta, can be obtained from an MD simulation of a system of $N$ atoms by diagonalizing the $3 N \times 3 N$ covariance matrix $\mathbf{K}$ of mass weighted cartesian velocities, whose elements are:

$$
K_{i j}=\frac{1}{2}\left\langle\sqrt{m_{i} m_{j}} v_{i}(t) v_{j}(t)\right\rangle
$$

where $m$ and $v$ indicate, respectively, atomic masses and (vibrational) velocities in the bodyfixed frame that translates and rotates with the system, and $i$ and $j$ run over the $3 N$ cartesian components. The matrix $\mathbf{L}$ whose columns are the $3 N$ normalized vibrational mode eigenvectors derived from diagonalization of $\mathbf{K}$ can be used to obtain a set of generalized normal mode velocities at each step of an MD trajectory by the following projection:

$$
\dot{\mathbf{Q}}(t)=\mathbf{L}^{\mathrm{T}} \mathbf{v}(t)
$$

where $\dot{\mathbf{Q}}(t)$ and $\mathbf{v}(t)$ are $3 N \times 1$ vectors of the instantaneous generalized normal mode and body-fixed-frame velocities, respectively, and $\mathbf{L}^{\mathrm{T}}$ is the transpose of the matrix $\mathbf{L}$. The vibrational kinetic energy of the system can be decomposed into contributions from individual vibrational modes according to:

$$
T(t)=\frac{1}{2} \sum_{i=1}^{3 N} \dot{Q}_{i}^{2}(t)=\sum_{i=1}^{3 N} T_{i}(t)
$$

Thus, one can monitor the evolution of the portion of total vibrational kinetic energy shared by each generalized normal mode during a trajectory propagation, by projecting the body- 
fixed-frame velocities along the vibrational mode vectors through (10) and then computing the $T_{i}(t)$ terms appearing in (11). This procedure provides a means to draw a qualitative picture of intramolecular energy flow in a complex system and was recently successfully applied to analyze $a b$ initio MD trajectories to investigate IVR processes in uracil. ${ }^{66}$ Moreover, the generalized normal mode analysis briefly illustrated here was also used in another study, in conjunction with QM/MM simulations of a metal ion in water to decompose solute-solvent thermal fluctuations in terms of vibrational modes to support the analysis of X-ray absorption measurements. ${ }^{67}$ In the present work, generalized normal modes and corresponding velocities were computed for the PtPOP complex from both $\triangle \mathrm{SCF}$ gas-phase and QM/MM trajectories. For the $\triangle \mathrm{SCF}-\mathrm{QM} / \mathrm{MM}$ trajectories, where it was not possible to separate out translation and overall rotation of the solute during the propagation, the body-fixed-frame velocities to be used in (9) were obtained from the cartesian velocity vectors $\dot{\mathbf{x}}_{\alpha}(t)$ by an a posteriori procedure. First of all, we required that the origin is at the center of mass of the molecule, i.e. $\sum_{\alpha}^{N} m_{\alpha} \mathbf{x}_{\alpha}(t)=0$ and $\sum_{\alpha}^{N} m_{\alpha} \dot{\mathbf{x}}_{\alpha}(t)=0$, to separate the translation. Afterwards, we applied a rigid rotation to align all frames to a reference structure:

$$
\mathbf{x}_{\alpha}^{\prime}(t)=\mathbf{R}(t) \mathbf{x}_{\alpha}(t)
$$

where the rotation matrix $\mathbf{R}(t)$ was computed using the Kabsch method, ${ }^{68}$ which minimizes the root mean squared deviation (RMSD) between the instantaneous structure $\mathbf{x}^{\prime}(t)$ and the reference frame. Finally, we assumed the overall rotational energy and internal kinetic energy of the molecule to be completely separable, such that the total kinetic energy is given by:

$$
E_{k}(t)=\frac{1}{2} \dot{\mathbf{X}}^{2}(t) \sum_{\alpha=1}^{N} m_{\alpha}+\frac{1}{2} \sum_{\alpha=1}^{N} m_{\alpha}\left(\boldsymbol{\omega}_{r}(t) \times \mathbf{x}_{\alpha}^{\prime}(t)\right)^{2}+\frac{1}{2} \sum_{\alpha=1}^{N} m_{\alpha} \mathbf{v}_{\alpha}^{2}(t)
$$

where $\mathbf{X}(t)$ is the translating position of the origin of the system of axis with respect to the fixed laboratory system and $\boldsymbol{\omega}_{r}(t)$ is the apparent angular velocity obtained from the instantaneous moment of inertia and angular momentum of the molecule $\left(\boldsymbol{\omega}_{r}(t)=\right.$ 
$\left.\mathbf{I}^{-1}(t) \sum_{\alpha}^{N} \mathbf{x}_{\alpha}^{\prime}(t) \times \dot{\mathbf{x}}_{\alpha}^{\prime}(t)\right)$; and calculated the body-fixed-frame velocities according to:

$$
\mathbf{v}_{\alpha}(t)=\dot{\mathbf{x}}_{\alpha}^{\prime}(t)-\boldsymbol{\omega}_{r}(t) \times \mathbf{x}_{\alpha}^{\prime}(t)
$$

When multiple trajectories were available, the average in equation (9) to compute the covariance matrix $\mathbf{K}$ was carried out over time and trajectories.

\section{Computational details}

In all simulations reported in this work, unless otherwise specified, the electronic structure of PtPOP was calculated using the GPAW DFT code. A frozen core approximation was employed in which the description of the core electrons is based on a reference scalar relativistic calculation of the isolated atoms (scalar relativistic effects are expected to be important in determining bond lengths involving Pt atoms ${ }^{69}$ ), and the KS orbitals for the valence electrons are represented in a basis of linear combination of atomic orbitals (LCAO). ${ }^{70}$ The excited states were described with the $\triangle \mathrm{SCF}$ scheme presented herein, with a $\sigma$ of $0.01 \mathrm{eV}$ for the Gaussian smearing of the orbital occupation numbers. This value of $\sigma$ was found to bring no detectable changes in the PESs of PtPOP calculated in vacuum, while at the same time allowed to readily converge all steps of all $\triangle \mathrm{SCF} B O M D$ trajectories. For the $\mathrm{S}_{1}$ state, the spin-unpolarized formalism was used by enforcing an initial occupation number of 1 for the HOMO and LUMO of PtPOP. This approach is computationally much cheaper in geometry optimizations and MD simulations than employing Ziegler's sum rule, ${ }^{40}$ because the latter requires SCF convergence of two single-determinant states, one having mixed singlet-triplet and one with triplet spin symmetry. Although spin-unpolarized $\Delta \mathrm{SCF}$ calculations lack a formal justification, their accuracy in estimating transition energies of transition metal complexes was shown to be superior, in some cases, to the approach based on the sum rule. ${ }^{45}$ This success was rationalized on the basis of similarities between the spin-unpolarized $\triangle \mathrm{SCF}$

density and an ensemble density. ${ }^{45,71}$ The exchange-correlation functional employed in the 
calculations was the GGA functional BLYP, ${ }^{72,73}$ while the basis functions were tzp ${ }^{70}$ for the Pt atoms and $\mathrm{dzp}^{70}$ for all other atoms. We used a grid spacing of the GPAW cell of 0.18 A. This choice of LCAO basis set and grid spacing ensures that the structure of the complex is converged with respect to these simulation parameters, as shown in the Supporting Information (SI).

The geometries of the ground state $\left(\mathrm{S}_{0}\right)$ and lowest singlet $\left(\mathrm{S}_{1}\right)$ and triplet $\left(\mathrm{T}_{1}\right)$ excited states of PtPOP were fully optimized in vacuum using a quasi-Newton local optimization algorithm implemented in ASE. A previous DFT study ${ }^{74}$ identified two conformers of PtPOP in the ground state with staggered $\left(D_{4}\right.$ symmetry) and eclipsed ( $C_{4 h}$ symmetry) hydrogen bonding motifs, respectively, the eclipsed structure being about $0.036 \mathrm{eV}$ more stable at the DFT-B3LYP level. In the present work, we optimized the more stable ground-state conformation; this structure was then used as a starting point to optimize the geometry in the excited states. Geometry optimization was carried out until the maximum force on all individual atoms was less than $0.02 \mathrm{eV} / \AA$. In addition to the geometry optimizations in ASE and GPAW, we performed, for comparison, geometry optimizations of the complex in the $\mathrm{S}_{0}$ and $\mathrm{T}_{1}$ states using a standard implementation of KS DFT within the Gaussian09 program package, ${ }^{75}$ and the unrestricted Kohn-Sham (UKS) approach for the excited state. These simulations employed the Ahlrichs TZVP ${ }^{76}$ all-electron basis set for the $\mathrm{P}, \mathrm{O}, \mathrm{H}$ atoms, and the quasirelativistic effective core potential (ECP) def2-ECP 77 in conjunction with the valence electrons Ahlrichs def2-TZVP ${ }^{78}$ basis set for the Pt atoms. Two different exchange-correlation functionals were used for these calculations: the BLYP functional, which was also utilized in the GPAW calculations, and the commonly employed hybrid functional B3LYP ${ }^{79,80}$ to test the effect of including a portion of exact Hartree-Fock exchange energy on the structure of the complex. All fully-optimized geometries in this work were confirmed to be true minima of the potential energy surface by inspection of the frequencies of normal mode (NM) analyses.

Potential energy curves in vacuum in a particular electronic state were computed by 
scanning along relevant coordinates, starting from the fully-optimized geometry of that state, while relaxing at each step all other degrees of freedom with the same convergence criteria as used in the full geometry optimizations in ASE.

As explained in the introduction, we performed $\triangle \mathrm{SCF}-\mathrm{QM} / \mathrm{MM}$ BOMD simulations to investigate the interplay between solvent effects and IVR. However, due to the vast amount and statistical variability of parallel processes playing out in solution, extracting clear indications about the most likely paths of energy relaxation from a MD-generated outof-equilibrium solution ensemble can be arduous if not impracticable at all. In fact, the interplay between anharmonic couplings and stochastic events can lead to incoherent processes, making the monitoring of average dynamical properties useless, while, at the same time, extrapolation of ensemble trends from the behaviour of a few individual uncorrelated trajectories can be dangerous, due to statistical bias. For this reason, we have performed additional $\triangle \mathrm{SCF}$ BOMD simulations of an isolated PtPOP molecule in $\mathrm{S}_{1}$ with the aim to gain preliminary insights into the excited-state intramolecular energy flow and, thus, facilitate the interpretation of the vibrational analysis of the $\triangle \mathrm{SCF}-\mathrm{QM} / \mathrm{MM}$ trajectories. The $\triangle \mathrm{SCF}$ BOMD simulations in vacuum were performed by propagating the system with Velocity Verlet with an integration time step of $1 \mathrm{fs}$. To allow a time step of $1 \mathrm{fs}$, all O-H bonds and hydrogen bonds present in the complex were constrained with the ASE implementation of RATTLE. ${ }^{81}$ This choice was found to negligibly affect the average structural and dynamical properties of PtPOP in the ground state. ${ }^{34}$ During the dynamics, the translational and rotational degrees of freedom (DOF) were removed at each step by projecting out the total linear and angular momenta, respectively.

For the $\triangle \mathrm{SCF}-\mathrm{QM} / \mathrm{MM}$ BOMD simulations we used the multiscale scheme implemented in ASE, ${ }^{34}$ which couples GPAW with classical potential functions to electrostatically embed the DFT-QM solute in a system of fixed point charges representing the solvent. The ultrafast dynamics taking place in water after photoexcitation to the $\mathrm{S}_{1}$ electronic state was approximated by propagating $\triangle \mathrm{SCF}-\mathrm{QM} / \mathrm{MM}$ trajectories starting from BOMD configu- 
rations selected from about 460 ps of $300 \mathrm{~K}$ thermally equilibrated ground-state QM/MM trajectories, which were collected in our previous work. ${ }^{34}$ A complete account of the MD protocol that was used to produce the QM/MM BOMD data for the ground state can be found in. ${ }^{34}$ Here we recapitulate the key settings of the QM/MM simulation setup. We used a cubic simulation box with side length of $35 \AA$. The MM region comprised 1383 TIP4P water molecules and $4 \mathrm{~K}^{+}$counterions to neutralize the total charge of the box. Non-bonded dispersion and exchange repulsion interactions were parametrized through the standard Lennard-Jones (LJ) potential, ${ }^{34}$ using for the atoms of the complex van der Waals parameters from the universal force field (UFF). ${ }^{82}$ Throughout the dynamics the positions of the counterions were restrained by applying a spherical harmonic potential of the form:

$$
V^{\mathrm{PR}}\left(\mathbf{x}_{i}\right)= \begin{cases}\frac{1}{2} k_{\mathrm{pr}}\left(d_{i}^{\prime}-d_{\mathrm{c}}^{\mathrm{pr}}\right)^{2} & \text { if } d_{i}^{\prime} \leq d_{\mathrm{c}}^{\mathrm{pr}} \\ 0 & \text { if } d_{i}^{\prime}>d_{\mathrm{c}}^{\mathrm{pr}}\end{cases}
$$

where $\mathbf{x}_{i}$ is the position vector of counterion $i$ and $d_{i}^{\prime}=\left|\mathbf{x}_{i}-\mathbf{x}^{\mathrm{CQM}}\right|$, with $\mathbf{x}^{\mathrm{CQM}}$ the center of the QM cell. The cutoff radius $d_{\mathrm{c}}^{\mathrm{pr}}$ and the force constant $k_{\mathrm{pr}}$ for the harmonic restraint potential were chosen to be equal to $16 \AA$ and $500 \mathrm{kcal} / \mathrm{mol}$ respectively. Propagation was done in the NVT ensemble at $300 \mathrm{~K}$ by applying the Langevin thermostat as implemented in $\mathrm{ASE}^{83}$ to the solvent molecules. All $\mathrm{O}-\mathrm{H}$ bonds and hydrogen bonds in the complex were constrained with RATTLE to achieve a time step of 2 fs. The simulations resulted in around 230000 equilibrated MD snapshots collected over a total simulation time of about 460 ps, which made up the equilibrium ensemble of ground-state configurations from which initial conditions for the nonequilibrium dynamics in the excited state were sampled. Freezing the $\mathrm{O}-\mathrm{H}$ bonds in the solute and the solvent does not undermine a trustworthy description of the relaxation of the solvation shell, as it is known ${ }^{84-86}$ that internal solvent vibrations have a negligible contribution in the relaxation process as compared to librational and translational motions. 
Excitation to the $\mathrm{S}_{1}$ state by an ultrashort optical pulse was simulated by instantaneously promoting ground-state molecules from the underlying ground-state distribution of Pt-Pt distances $\left(P_{\mathrm{GS}}^{\mathrm{eq}}\left(d_{\mathrm{PtPt}}\right)\right)$ according to a spatial filtering $(\mathrm{SF})$ approximation. ${ }^{87-91}$ This approximation takes into account the bandwidth of the pulse but neglects any effect due to nuclear motion throughout its finite temporal duration. Hence, we assumed a Gaussian ultrashort pulse $\epsilon(t) \propto \mathrm{e}^{-\frac{t^{2}}{2 \tau^{2}}} \mathrm{e}^{-i \omega_{1} t}$, where $\omega_{1}$ and $\tau$ are respectively the center frequency and temporal width, and sampled initial conditions for a set of excited-state trajectories from the (unnormalized) distribution $P_{\mathrm{ES}}\left(d_{\mathrm{PtPt}}, t_{0}\right)$ given by:

$$
P_{\mathrm{ES}}\left(d_{\mathrm{PtPt}}, t_{0}\right)=F^{2}\left(d_{\mathrm{PtPt}}\right) P_{\mathrm{GS}}^{\mathrm{eq}}\left(d_{\mathrm{PtPt}}\right)
$$

In equation (16) the excitation window $F\left(d_{\mathrm{PtPt}}\right)$ takes the form:

$$
F\left(d_{\mathrm{PtPt}}\right) \propto \exp \left[-\frac{\tau^{2}\left(\Delta V\left(d_{\mathrm{PtPt}}\right)-\hbar \omega_{1}\right)^{2}}{2 \hbar^{2}}\right]
$$

where $\Delta V\left(d_{\mathrm{PtPt}}\right)$ is the potential energy difference between the ground and excited states. An extensive discussion about the approximations underlying the SF approximation and a comparison with other methods to select initial conditions for the excitation process are provided in the Supporting Information. The parameters for the excitation pulse were chosen as those of the pulse used in the transient absorption setup by van der Veen et al. ${ }^{14}$ to probe the ultrafast vibrational dynamics upon excitation into $\mathrm{S}_{1}$ of PtPOP in water. $\omega_{1}$ corresponds approximately to the position of the maximum of the experimental absorption spectrum of PtPOP in water, which is at $\sim 370 \mathrm{~nm},{ }^{50,52}$ giving a $\hbar \omega_{1}$ of $3.35 \mathrm{eV}$; while $\tau$ was $60 \mathrm{fs}$ (corresponding to a full width at half maximum (FWHM) of the Gaussian intensity profile of $\sim 100 \mathrm{fs}) .{ }^{14} \Delta V\left(d_{\mathrm{PtPt}}\right)$ was taken as the difference between Morse potentials obtained from a fit to the potentials of mean force (PMF) calculated from the pairwise Pt-Pt radial 
distribution functions (RDFs) for $\mathrm{S}_{0}$ and $\mathrm{S}_{1}$ as:

$$
\mathrm{w}\left(d_{\mathrm{PtPt}}\right)=-k_{b} T \ln \left(g_{\mathrm{PtPt}}\left(d_{\mathrm{PtPt}}\right)\right)
$$

where $g_{\mathrm{PtPt}}\left(d_{\mathrm{PtPt}}\right)$ is the Pt-Pt RDF of the ground or excited state. For the ground state $g_{\mathrm{PtPt}}\left(d_{\mathrm{PtPt}}\right)$ was obtained from the $460 \mathrm{ps}$ of equilibrated QM/MM BOMD data previously collected for $\mathrm{S}_{0}$, and the relative PMF was reported in; ${ }^{34}$ for the excited state 50 preliminary $\mathrm{S}_{1} \triangle \mathrm{SCF}-\mathrm{QM} / \mathrm{MM}$ trajectories were produced starting from as many ground-state frames with a Pt-Pt distance close to the Pt-Pt distance of the $\mathrm{S}_{1}$ optimized geometry. The trajectories were run with a time step of $2 \mathrm{fs}$, keeping the thermostat applied to the solvent, and summed up, at the end of the propagation, to around $200 \mathrm{ps}$, from which $g_{\mathrm{PtPt}}\left(d_{\mathrm{PtPt}}\right)$ was calculated $\left(g_{\mathrm{PtPt}}\left(d_{\mathrm{PtPt}}\right)\right.$ was characterized by an average Pt-Pt distance and width that were found to be the same as for $g_{\mathrm{PtPt}}\left(d_{\mathrm{PtPt}}\right)$ obtained by removing the non-equilibrated part of the trajectories, the two RDFs differing only by the level of statistical noise). Finally, the two potentials were shifted relative to each other such that the energy difference at the average $d_{\mathrm{PtPt}}$ of the equilibrium ground-state distribution was equal to $3.35 \mathrm{eV}$, i.e. the transition energy at the maximum of the $S_{0} \rightarrow S_{1}$ band of the experimental absorption spectrum (and center frequency of the experimental pulse ${ }^{14}$ ). Since the simulated average $\mathrm{S}_{0} \rightarrow \mathrm{S}_{1}$ transition energy in solution was found to be $3.24 \mathrm{eV}$ (see Table 1 in the Supporting Information), this implied that the $\mathrm{S}_{1}$ potential had to be shifted upwards by $0.11 \mathrm{eV}$. Once $P_{\mathrm{ES}}\left(d_{\mathrm{PtPt}}, t_{0}\right)$ was obtained according to (16) and (17), $49 \mathrm{~S}_{1} \Delta \mathrm{SCF}-\mathrm{QM} / \mathrm{MM}$ trajectories were started from configurations of the ground-state equilibrium ensemble giving a distribution of Pt-Pt distances reflecting $P_{\mathrm{ES}}\left(d_{\mathrm{PtPt}}, t_{0}\right)$. In the selection of ground-state frames we ensured that they where spaced at least $0.5 \mathrm{ps}$ from each other, such to minimize the correlation between them. The ultrafast relaxation in the $\mathrm{S}_{1}$ state of PtPOP following preparation of the nonequilibrium ensemble $P_{\mathrm{ES}}\left(d_{\mathrm{PtPt}}, t_{0}\right)$ by laser promotion is provided by the first $\sim 2.5 \mathrm{ps}$ of this set of trajectories. Equilibrium solute-solvent RDFs for $\mathrm{S}_{1}$ and related 
properties were obtained from the two sets of excited-state trajectories after removing the first (nonequilibrated) $2.5 \mathrm{ps}$ from each of them, which gave a total of around 80000 MD snapshots covering 160 ps.

\section{Results and discussion}

\section{Molecular geometry from vacuum calculations}

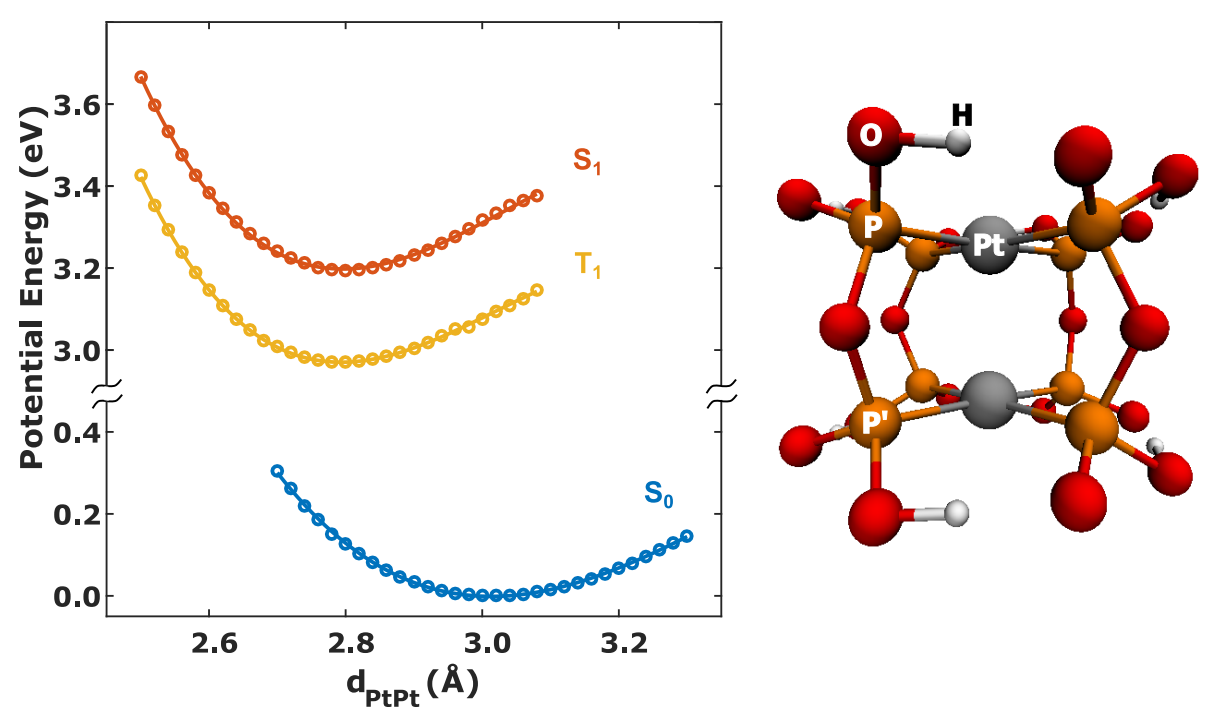

Figure 1: (Left) PESs along the Pt-Pt coordinate computed in vacuum for the $\mathrm{S}_{0}, \mathrm{~S}_{1}$ and $\mathrm{T}_{1}$ states of PtPOP. Open circles represent the calculated points, while the lines are 3rd order polynomial fits. (Right) Visualization of the PtPOP complex with the atomic labels used to indicate the structural parameters reported in Table 1. The molecular structure represented here corresponds to the geometry fully optimized in the ground state. The PESs and the optimized geometry were obtained from vacuum calculations using GPAW with the BLYP functional.

Relevant structural parameters of the $\mathrm{S}_{0}$ state of PtPOP (see Figure 1 (right) for a depiction of the molecule) together with the differences with respect to the $S_{1}$ and $T_{1}$ structures obtained from the geometry optimizations in vacuum are given in Table 1. The ground state is found to have approximate $C_{4 h}$ symmetry, with a $D_{4 h} \mathrm{Pt}_{2} \mathrm{P}_{8}$ core. The largest discrepancy between the $\mathrm{S}_{0}$ structure predicted using GPAW and the one obtained using more conventional atom-centered basis sets and an ECP for Pt with the same exchange-correlation 
Table 1: Selected structural parameters for the $\mathbf{S}_{0}, \mathrm{~S}_{1}$ and $\mathbf{T}_{1}$ states of PtPOP obtained from the geometries optimized in vacuum at different DFT levels

\begin{tabular}{|c|c|c|c|c|c|c|c|}
\hline & \multicolumn{3}{|c|}{$\mathrm{S}_{0}$} & \multicolumn{3}{|c|}{$\Delta\left(\mathrm{T}_{1}-\mathrm{S}_{0}\right)^{b}$} & \multirow{3}{*}{$\begin{array}{c}\Delta\left(\mathrm{S}_{1}-\mathrm{S}_{0}\right) \\
\text { BLYP } \\
\text { GPAW }\end{array}$} \\
\hline & \multirow[b]{2}{*}{$\begin{array}{l}\text { BLYP } \\
\text { GPAW }\end{array}$} & \multicolumn{2}{|c|}{ def2-ECP/TZVP ${ }^{a}$} & \multirow[b]{2}{*}{$\begin{array}{l}\text { BLYP } \\
\text { GPAW }\end{array}$} & \multicolumn{2}{|c|}{ def2-ECP /TZVP ${ }^{a}$} & \\
\hline & & BLYP & B3LYP & & BLYP & B3LYP & \\
\hline \multicolumn{8}{|l|}{ Bond $(\AA)$} \\
\hline $\mathrm{Pt}-\mathrm{Pt}$ & 3.005 & 3.091 & 3.065 & -0.211 & -0.241 & -0.248 & -0.205 \\
\hline Pt-P & 2.393 & 2.425 & 2.399 & 0.031 & 0.032 & 0.019 & 0.032 \\
\hline $\mathrm{P}-\mathrm{O}\left(-\mathrm{P}^{\prime}\right)$ & 1.718 & 1.711 & 1.679 & 0.001 & 0.002 & -0.000 & 0.001 \\
\hline $\mathrm{P} \cdots \mathrm{P}^{\prime}$ & 3.098 & 3.126 & 3.084 & -0.060 & -0.062 & -0.067 & -0.055 \\
\hline \multicolumn{8}{|l|}{ Angles (deg) } \\
\hline $\mathrm{P}-\mathrm{O}-\mathrm{P}^{\prime}$ & 128.84 & 131.90 & 133.38 & -4.63 & -5.07 & -5.39 & -4.27 \\
\hline$(\mathrm{Pt}-\mathrm{Pt}-\mathrm{P})_{\alpha}$ & 91.14 & 90.40 & 90.23 & 5.49 & 5.45 & 4.42 & 5.42 \\
\hline$(\mathrm{Pt}-\mathrm{Pt}-\mathrm{P})_{\beta}$ & 91.08 & 90.42 & 90.22 & -1.96 & -1.28 & -0.14 & -1.93 \\
\hline P-Pt-Pt-P' & 0.03 & 0.00 & 0.01 & 0.61 & 0.54 & 0.36 & 0.46 \\
\hline
\end{tabular}

functional, is in the Pt-Pt distance, which is $0.086 \AA$ shorter in the GPAW structure. We note that the GPAW calculated Pt-Pt distance of $3.005 \AA$ is much closer to the experimental range (2.913-2.979 $\AA$ ) of values found from X-ray crystallography. ${ }^{55,92-94}$ The differences become smaller in the excited states since the structure calculated with standard KS DFT experiences a larger Pt-Pt contraction. The $\mathrm{S}_{1}$ and $\mathrm{T}_{1}$ excited states are formed by promotion of an electron from the metal-metal $\mathrm{d} \sigma^{*} \mathrm{HOMO}$ antibonding to the metal-metal $\mathrm{p} \sigma$ LUMO bonding orbital of the complex. Eventually, the excitation results in the formation of a bond between the two Pt atoms with consequent shortening of the Pt-Pt distance. The Pt-Pt contractions from ground to excited state predicted by all different methods are well within the experimental range (0.19-0.28 $\AA$ ) of values obtained from Franck-Condon analysis of the vibronic progression of low-temperature absorption and emission spectra ${ }^{51,57}$ and X-ray diffraction measurements ${ }^{55,56}$ of crystals. The Pt-Pt bond in the $\mathrm{T}_{1}$ state is found to be shorter than in the $\mathrm{S}_{1}$ state of $\sim 0.01 \AA$ from the GPAW calculations. Indeed, a slightly reduced contraction in the singlet excited state with respect to the triplet has been inferred experimentally by comparing the wavenumbers of the Pt-Pt stretching progression exhibited 
by the absorption bands of crystal $\left(\mathrm{n}-\mathrm{Bu}_{4} \mathrm{~N}\right)_{4}[\mathrm{PtPOP}]$ relative to the $\mathrm{S}_{1}\left(145-147 \mathrm{~cm}^{-1}\right)$ and $\mathrm{T}_{1}\left(150 \mathrm{~cm}^{-1}\right)$ states, $^{7,50}$ and was further confirmed by the DFT calculations performed by Záliš et al. ${ }^{58}$ using the PBE0 functional, which delivered a $\Delta\left(\mathrm{S}_{1}-\mathrm{T}_{1}\right)$ for the Pt-Pt bond of $\sim 0.02 \AA$. In Figure 1 (left) we report the PESs computed along the Pt-Pt coordinate for all three electronic states using GPAW with the BLYP functional. As expected, the PESs of $\mathrm{T}_{1}$ and $\mathrm{S}_{1}$ are shifted to shorter Pt-Pt distances with respect to the ground state and parallel to each other, a feature that, up to now, had only been postulated experimentally based on the similarities of the low-temperature $S_{0} \rightarrow S_{1}$ and $S_{0} \rightarrow T_{1}$ absorption bands. ${ }^{7,50}$ To our knowledge, this is the first time that this experimental observation is confirmed by a DFT calculation of the $\mathrm{S}_{1}$ and $\mathrm{T}_{1}$ PESs of PtPOP. Inspection of the relative energies of the singlet and triplet excited states from Figure 1 reveals that the simulations underestimate the experimental single-triplet splitting by a factor of around 2.5. The $\mathrm{S}_{1}$ vertical transition energy calculated at the $\mathrm{S}_{0}$ optimized geometry by the $\triangle \mathrm{SCF}$ method is $3.51 \mathrm{eV}$, in good agreement with the position of the maximum of the absoption spectra of PtPOP between 360 and $370 \mathrm{~nm}(3.35-3.44 \mathrm{eV})$ for different crystals. ${ }^{50,51}$ The discrepancy in the singlet-triplet splitting is due to the triplet excited state being understabilized by $\sim 0.5 \mathrm{eV}$ in the calculations. A similar excitation energy for the triplet was obtained by Novozhilova et al. ${ }^{59}$ by TDDFT with the BLYP functional and an all-electron basis set for Pt. Thus, the failure in correctly reproducing the energy of $\mathrm{T}_{1}$ might come from deficiencies of the GGA functional BLYP in properly accounting for the exchange repulsion term in the $\mathrm{T}_{1}$ state of PtPOP. However, since the present work focuses exclusively on structural properties of the molecule and on the $\mathrm{BO}$ dynamics in the $\mathrm{S}_{1}$ state that occurs at times considerably shorter than the ISC times observed for PtPOP in water solution, reproducing an accurate energy picture of the lowest triplet excited state was not relevant for this study.

Turning to the other geometrical parameters, the most prominent changes between ground- and excited-state structures in interatomic distances involving atoms in the ligands are represented by a lengthening of the Pt-P bonds and by a shortening of the $\mathrm{P} \cdots \mathrm{P}^{\prime}$ distan- 
ces along the Pt-Pt axis, which is, however, much smaller than the Pt-Pt contraction itself. An elongation of the Pt-P bonds in the excited state of PtPOP is a well-known prediction of DFT. ${ }^{59}$ Since using B3LYP results in a $\sim 40 \%$ smaller elongation, as evident from Table 1, it seems also to be the structural effect of excitation that is most sensible to the introduction of exact exchange in the DFT functional. Apart from that, BLYP and B3LYP predicted structural changes from ground to excited state agree within $0.007 \AA$ for bond lengths and $1^{\circ}$ for angles, while differences in the ground state are all smaller than $2 \%$ of the BLYP calculated values. Therefore, given the similarities between BLYP and B3LYP results in this case, it was possible to perform BOMD simulations using the computationally cheaper GGA functional without loss of accuracy with respect to DFT with a hybrid functional.

An interesting aspect of the optimized geometry of the excited states that emerges from inspection of the angles reported in Table 1 is that $\mathrm{PtP}_{4}$ moieties do not retain a local square based planar geometry but slightly distort towards a quasi-trigonal bipyramidal structure (see also Figure 2). This results in a lowering of the symmetry of the $\mathrm{Pt}_{2} \mathrm{P}_{8}$ core of the molecule from $D_{4 h}$ to $D_{2 d}$. The extent of the distortion can be quantified by the difference (indicated by $\Delta$ ) between $\angle \mathrm{Pt}-\mathrm{Pt}-\mathrm{P}$ angles involving equatorial and axial $\mathrm{P}$ atoms of the local quasi-trigonal bipyramidal geometry (indicated by $\alpha$ and $\beta$, see Figure 2). To characterize in more detail this structural distortion involving the ligands, we have computed the PES in the $\mathrm{S}_{1}$ state along the coordinate $\Delta$. The PES is shown in Figure 2 and clearly reveals the presence of a rotational barrier between equivalent $D_{2 d}$ geometries. The pseudorotation of the $\mathrm{P}$ atoms in each $\mathrm{PtP}_{4}$ group resembles the Berry isomerization mechanism ${ }^{95}$ occurring in trigonal bipyramidal molecules, although the angle $\angle(\mathrm{Pt}-\mathrm{Pt}-\mathrm{P})_{\alpha}$ does not reach the $120^{\circ}$ value characteristic of a perfect bipyramidal geometry due to the rigidity of the P-O-P bridging ligands. $D_{2 d}$ isomers of transition metal $\mathrm{M}_{2} \mathrm{~L}_{8}$ dimers, where each $\mathrm{ML}_{4}$ is in a local trigonal bipyramidal geometry and can undergo Berry pseudorotation, are known, ${ }^{96}$ but have never been reported before for PtPOP. From the experimental side, Ohashi and co-workers ${ }^{55}$ interpreted the outcome of time-resolved X-ray diffraction measurements of 


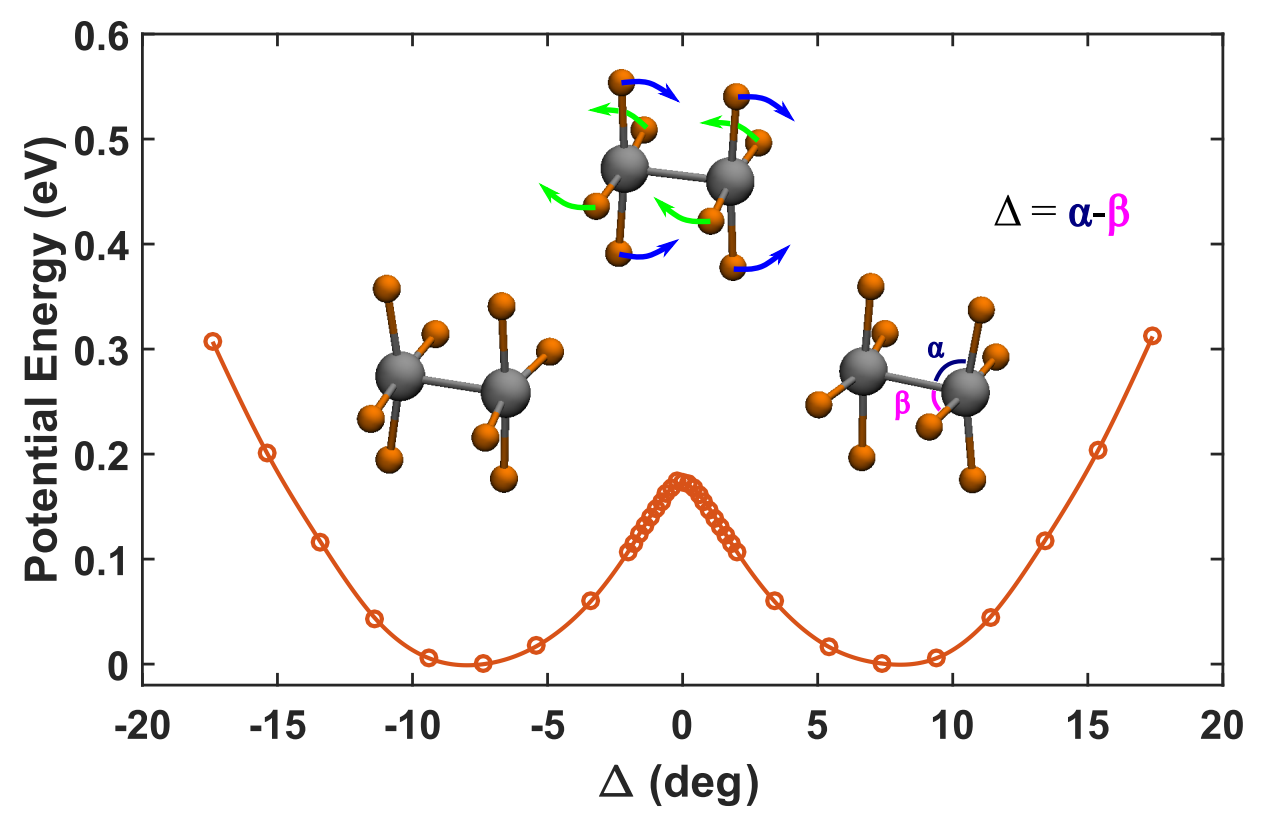

Figure 2: Vacuum PES of the $\mathrm{S}_{1}$ state of PtPOP along the pseudorotation coordinate $\Delta$. $\Delta$ is the angle difference defined in the figure. The $\mathrm{Pt}_{2} \mathrm{P}_{8}$ core of $\mathrm{PtPOP}$ is shown at the symmetric minima and at the transition state of the potential energy curve. The structure at $\Delta=0$ has $D_{4 h}$ symmetry and each $\mathrm{PtP}_{4}$ group is in a local square pyramidal geometry, as in the fully optimized ground-state molecule. The minima correspond to a $\mathrm{Pt}_{2} \mathrm{P}_{8}$ core with $D_{2 d}$ symmetry and $\mathrm{PtP}_{4}$ groups in a quasi-trigonal bipyramidal geometry. Open circles represent the calculated points, while the line is a cubic spline fit to the data.

crystals assuming $D_{4 h}$ symmetry. However, the analysis derived a large contraction of $\sim$ 0.1-0.2 $\AA$ of the Pt-P bonds, which is in contrast to the slight lengthening obtained from all DFT calculations. Moreover, it should be considered that in crystals there are packing forces and interactions with counterions that might come into play, which are not taken into account in the calculations of the gas-phase isolated molecule, making the validity of a direct comparison with experiments dubious. It is difficult, on the other hand, to explain why previous computational works where the structure of PtPOP in the triplet state was optimized with unrestricted DFT without symmetry constraints, have not reported this ligand distortion with symmetry lowering of the $\mathrm{Pt}_{2} \mathrm{P}_{8}$ core. The existence of a local minimum at a geometry with $D_{2 d}$ symmetry for both the $\mathrm{T}_{1}$ and $\mathrm{S}_{1}$ states is supported by all type of DFT calculations presented here, and was reproduced also by a GGA functional different than BLYP, as shown in Figure S5 of the Supporting Information. In the absence 
of detailed information about the true nature of reported $\mathrm{T}_{1}$ geometries in the literature, we speculate that this ligand distortion might have been overlooked.

\section{Equilibrium solution structure}

Table 2: Structural parameters of PtPOP in water obtained as averages over equilibrium QM/MM BOMD data for the $S_{0}$ and $S_{1}$ states of PtPOP, and comparison with available solution experimental values ${ }^{a}$. The MD average was carried out over a total simulation time of $\sim 460 \mathrm{ps}$ for $S_{0}$ and of $\sim 160 \mathrm{ps}$ for $S_{1}$.

\begin{tabular}{|c|c|c|c|c|c|}
\hline & \multicolumn{2}{|c|}{$\mathrm{S}_{0}$} & \multicolumn{2}{|c|}{$\Delta\left(\mathrm{S}_{1}-\mathrm{S}_{0}\right)$} & \multirow{2}{*}{$\frac{\Delta\left(\mathrm{T}_{1}-\mathrm{S}_{0}\right)}{\operatorname{Exp}^{53,54}}$} \\
\hline & Calc $^{34}$ & $\operatorname{Exp}^{54,97}$ & Calc & $\operatorname{Exp}^{98}$ & \\
\hline Bond $(\AA)$ & & & & & \\
\hline Pt-Pt & 2.99 & $2.98^{b}$ & -0.20 & $-0.24(4)^{b}$ & $-0.24(6)^{b}$ \\
\hline Pt-P & 2.33 & $2.32(4)^{c}$ & 0.01 & - & $0.010(6)^{c}$ \\
\hline $\mathrm{P} \ldots \mathrm{P}^{\prime}$ & 3.09 & $2.92^{b}$ & -0.01 & - & $0.00(8)^{b}$ \\
\hline Angles (deg) & & & & & \\
\hline$(\mathrm{Pt}-\mathrm{Pt}-\mathrm{P})_{\alpha}$ & 91.2 & - & 5.0 & - & - \\
\hline$(\mathrm{Pt}-\mathrm{Pt}-\mathrm{P})_{\beta}$ & 91.2 & - & -0.3 & - & - \\
\hline
\end{tabular}

${ }^{a}$ As explained in the text, it is assumed to be possible to directly compare simulation results for $S_{1}$ to experimental values obtained for $T_{1}$ when experimental data for $S_{1}$ are not available. ${ }^{b}$ Obtained in water by X-ray scattering experiments. ${ }^{54,98}{ }^{c}$ Obtained in ethanol by X-ray absorption measurements. ${ }^{53,97}$

Table 2 reports bond lengths and angles obtained as averages over the thermally equilibrated $\mathrm{S}_{0}$ and $\mathrm{S}_{1} \mathrm{QM} / \mathrm{MM}$ trajectories in water. The only structural parameter of the $\mathrm{S}_{1}$ state of PtPOP in aqueous solution that has ever been determined experimentally is the Pt-Pt distance. This bond length was recently deduced from a fit to XFEL difference scattering data by Biasin et al. ${ }^{98}$ As seen in Table 2, the Pt-Pt distance predicted by the $\triangle \mathrm{SCF}-\mathrm{QM} / \mathrm{MM}$ BOMD simulations agrees to a very good extent with the experimental S1 Pt-Pt distance. For the other structural parameters in S1, there are no values available from experiments. On the other hand, structural parameters other than the Pt-Pt distance have been determined experimentally for the $\mathrm{T}_{1}$ state in solution, and are reported in Table 2. The latter include the $\mathrm{P} \cdots \mathrm{P}^{\prime}$ distances obtained by time-resolved X-ray scattering measurements by Christensen et al., ${ }^{54}$ and the Pt-P bond lengths derived by van der Veen et 
al. ${ }^{53,97}$ from a fit to time-resolved X-ray absorption spectra in ethanol. The $\mathrm{S}_{1}$ calculated structural parameters and the experimental values for $T_{1}$ agree within the uncertainties of the experiments. Assuming that the solution structures of the $\mathrm{S}_{1}$ and $\mathrm{T}_{1}$ states are virtually the same, as they are in gas-phase (see Table 1), the comparison can be taken as a validation of the $\triangle \mathrm{SCF}-\mathrm{QM} / \mathrm{MM}$ BOMD simulations with respect to structural parameters of PtPOP in $\mathrm{S}_{1}$, or as an indication that $\mathrm{S}_{1}$ and $\mathrm{T}_{1}$ indeed have the same structure. From a comparison with the corresponding structural parameters of the gas-phase optimized geometries, we notice that the average Pt-Pt distance in solution is only $0.01 \AA$ shorter, while the solvent affects much more significantly structural parameters involving ligand atoms. This is particularly evident for the Pt-P bonds, which in the ground state are found to be $\sim 0.06$ $\AA$ shorter than in the isolated geometry-optimized structure and in $\mathrm{S}_{1}$ experience a $\sim 70 \%$ smaller elongation. In addition, despite the fact that the shortening of the Pt-Pt distance due to excitation is found to be the same in vacuum and solution, the $\mathrm{P}$ atoms follow the $\mathrm{Pt}$ atoms in the contraction along the Pt-Pt axis to only $0.01 \AA, \sim 80 \%$ less than in gas phase. As a side note, we point out that differences induced by the presence of the solvent on these structural parameters of PtPOP are larger than the changes brought by the use of a hybrid DFT functional like B3LYP, as can be seen by comparing the values reported for the Pt-P and $\mathrm{P} \cdots \mathrm{P}^{\prime}$ distances in Table 1 and 2 . This, again, means that there would be no significant advantage in employing the computationally more expensive B3LYP functional instead of BLYP in the QM/MM BOMD simulations.

An analysis of the average values of the $\angle \mathrm{Pt}-\mathrm{Pt}-\mathrm{P}$ angles reveals that also in solution the $\mathrm{PtP}_{4}$ units are distorted towards a quasi-trigonal bipyramidal local geometry with respect to the ground state (though the angle difference $\Delta$ found in solvent is $\sim 5^{\circ}$, around $2^{\circ}$ smaller than for the optimized $\mathrm{S}_{1}$ vacuum geometry). This is an important result, because it hints at the fact that a direct $\mathrm{S}_{1} \rightarrow \mathrm{T}_{1}$ ISC mechanism might be active in solution, which could explain the $\sim 3000$-times faster ISC rates exhibited by PtPOP with respect to its perfluoroborated analogue, ${ }^{7,60}$ where pseudorotation of the bulkier and more rigid ligands 
is less likely. Indeed, the role of structural distortions in lowering the $D_{4 h}$ symmetry of the $\mathrm{Pt}_{2} \mathrm{P}_{8}$ core of PtPOP, thus promoting direct $\mathrm{SOC}$ between $\mathrm{S}_{1}$ and $\mathrm{T}_{1}$, has been often hypothesized but so far never proven. ${ }^{7,10,58,60}$

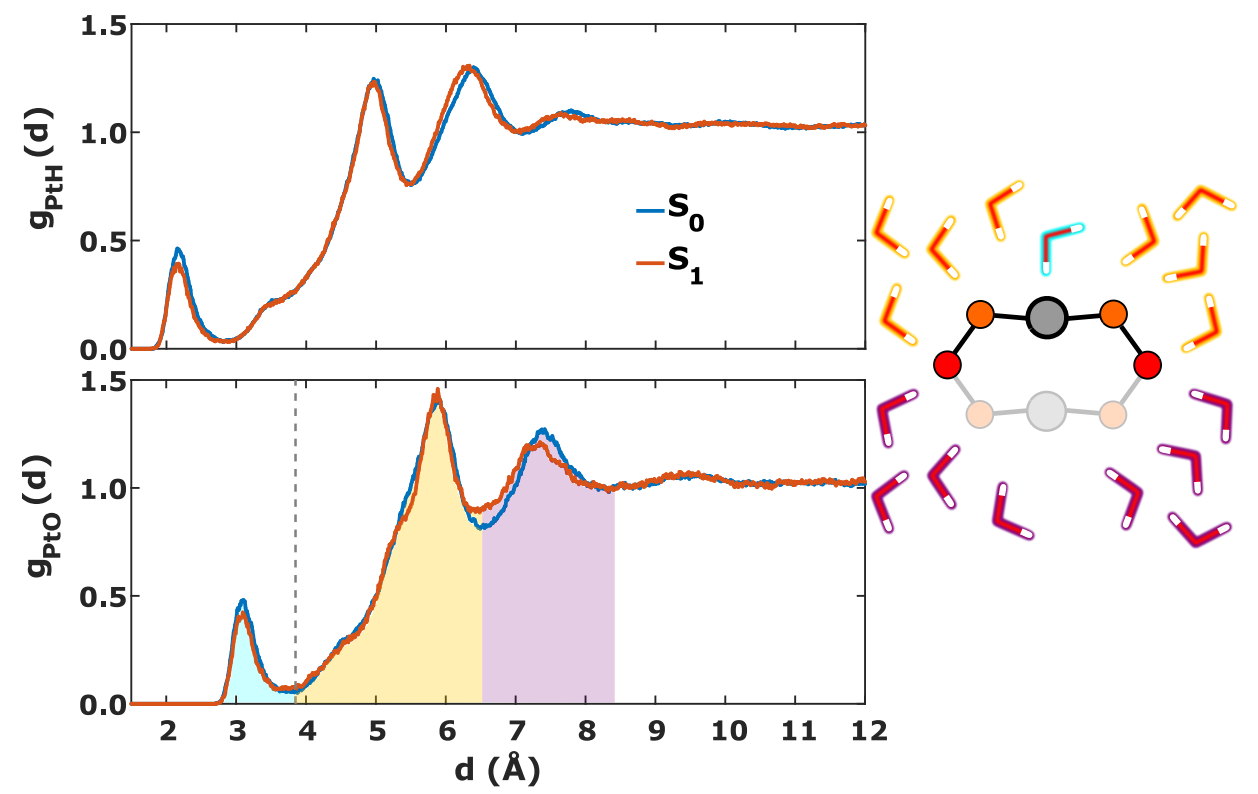

Figure 3: (Left) Pairwise Pt-solvent RDFs sampled from the equilibrated part of the $\mathrm{QM} / \mathrm{MM}$ trajectories in $\mathrm{S}_{0}$ and $\mathrm{S}_{1}$. The gray vertical line indicate the extent of the first solvation shell around one Pt atom. (Right) Illustration of the coordination of water molecules around a single Pt atom of PtPOP. Water molecules highlighted with a particular color belong to the peak of the Pt- $\mathrm{O}_{\text {solvent }} \mathrm{RDFs}$ with a shaded area of the same color.

We now turn to an examination of the solvation shell structure in the $\mathrm{S}_{1}$ state as compared to the one obtained for the ground state in our previous QM/MM BOMD study. ${ }^{34}$ Figure 3 shows the $\mathrm{Pt}-\mathrm{H}_{\text {solvent }}$ and $\mathrm{Pt}-\mathrm{O}_{\text {solvent }}$ RDFs extracted from the equilibrated trajectories in $\mathrm{S}_{1}$ and $\mathrm{S}_{0}{ }^{34}$ using a bin size of $0.01 \AA$ for the radial sampling. The position of water molecules within each of the coordination peaks with respect to a single Pt atom is illustrated schematically in Figure 3 (Right). The first peaks are indicative of the presence of strong $\mathrm{H}$-coordination of solvent molecules at the Pt-ends of the complex ${ }^{34}$ (molecules highlighted in blue in Figure 3 (Right)). The second coordination peaks (yellow water molecules) span $\mathrm{Pt}-\mathrm{H}_{\text {solvent }}$ distances between $\sim 4.5 \AA$ and $\sim 5.5 \AA$ and $\mathrm{Pt}-\mathrm{O}_{\text {solvent }}$ distances between $\sim 5.5 \AA$ and $\sim 6.5 \AA$. These peaks comprise water molecules that are found to lie mainly off-axis with respect to the $\mathrm{Pt}-\mathrm{Pt}$ direction. Due to the presence of two $\mathrm{Pt}$ atoms, and given the 
symmetry of the complex, water molecules of the second peaks make up also the third peaks of the RDFs $\left(5.5 \AA<d_{\mathrm{PtH}}<7.0 \AA\right.$ and $\left.6.5 \AA<d_{\mathrm{PtO}}<8.3 \AA\right)$. This is better illustrated by the water molecules highlighted in purple in the schematics of Figure 3 (Right). That water molecules belonging to the second (and third) peaks do not take up the space along the Pt-Pt direction is supported by the fact that the distance between the second and third peaks $(\sim 1.5 \AA)$ is less than the intramolecular Pt-Pt distance. In the excited state, neither the position of the first nor the second peaks change, which means that molecules in the two peaks must draw closer in conjunction with the $\mathrm{Pt}-\mathrm{Pt}$ contraction. This is further supported by a shift of the third peaks to shorter distances as this results from the Pt atoms finding themselves closer to water molecules located on the opposite sides of the complex in the excited state. The second most notable change in the RDFs is represented by a slightly reduced coordination in the first peaks. If the extent of the first coordination shell is taken up to the first minimum of the Pt- $\mathrm{O}_{\text {solvent }} \mathrm{RDF}$, at $3.85 \AA$, we can quantify the coordination with the running coordination number at this distance. It follows that the Pt- $\mathrm{O}_{\text {solvent }}$ coordination number in the first shell is found to be around 0.77 for PtPOP in $\mathrm{S}_{1}$, only $\sim 0.1$ smaller than in the ground state. Water molecules in this shell seem also to retain, after excitation, a preferential axial orientation, where the $\mathrm{O}-\mathrm{H}$ donor bond tends to point along the Pt-Pt axis of the solute. This is revealed by Figure 4, which shows that distributions of key solute-solvent angles, indicative of the extent of axial coordination, are largely unaltered by the excitation. Experimentally, emission spectra of PtPOP are found to be independent of the solvent. ${ }^{99}$ The finding that electronic excitation does not lead to any major restructuring in the local organization of solvent molecules surrounding the complex is in agreement with this experimental observation and points to the fact that this might be the case also for other types of solvents. This behaviour is in sharp contrast to the solvent shell response observed for photoexcitation of the $\mathrm{d}^{8}-\mathrm{d}^{8}$ complex $\left[\operatorname{Ir}_{2}(\operatorname{dimen})_{4}\right]^{2+}$ (where dimen is diisocyano-para-menthane) by ultrafast X-ray scattering measurements in acetonitrile. ${ }^{12}$ In that case, the effect of electronic excitation was found to be a loss of coordination of 


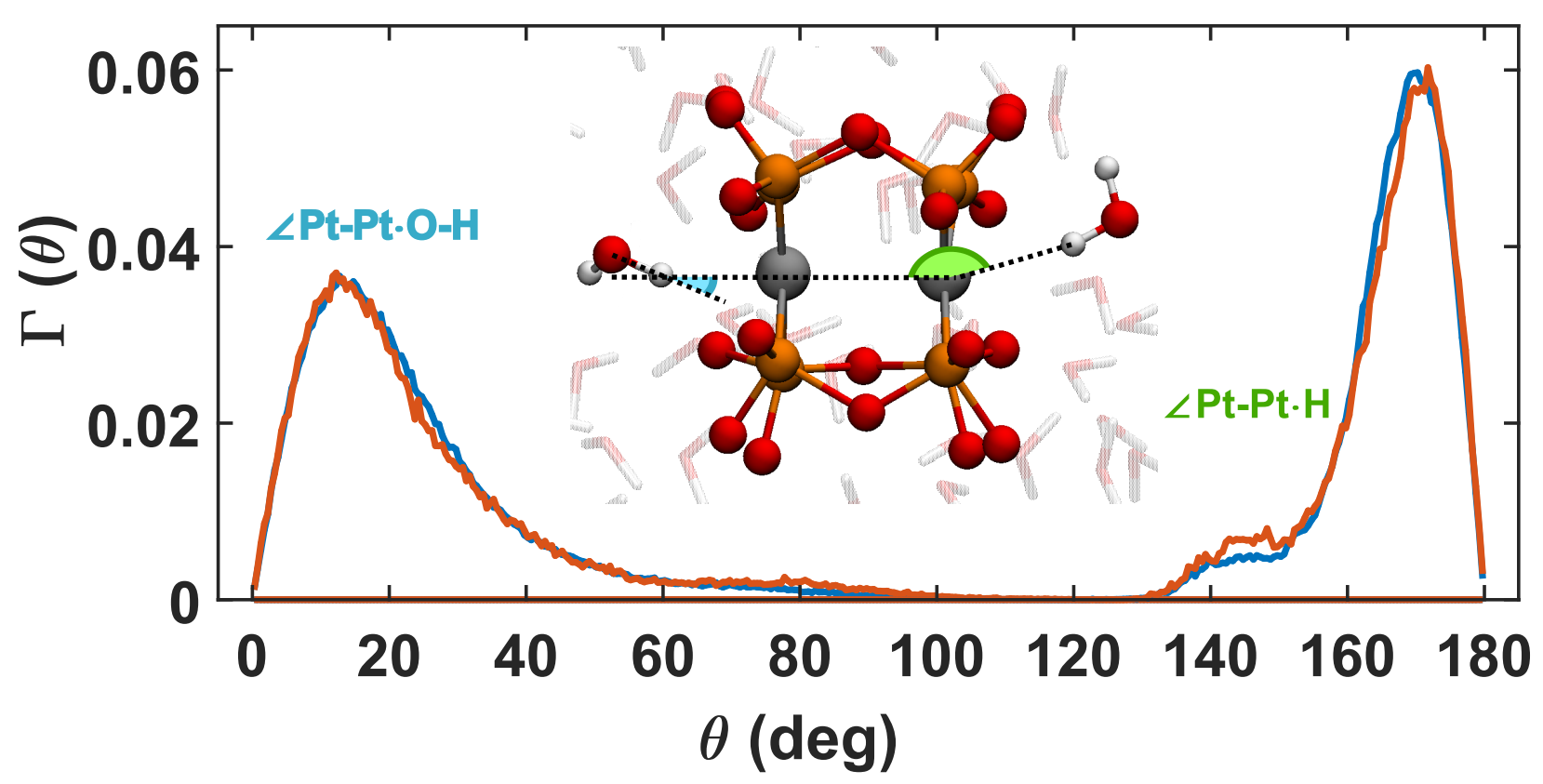

Figure 4: Probability distributions $(\Gamma)$ of solute-solvent angles involving water molecules in the first coordination shell of the Pt atoms in PtPOP as defined by the extent of the first peak of the $\mathrm{Pt}-\mathrm{O}_{\text {solvent }} \mathrm{RDF}$ in Figure 3. The color code for the distributions is the same as in Figure 3. The sampled angles are shown schematically using an MD snapshot selected from one of the $\mathrm{S}_{1} \Delta \mathrm{SCF}-\mathrm{QM} / \mathrm{MM}$ trajectories (for visualization purposes hydrogen atoms of the solute are omitted), and are related to the orientation of water molecules with respect to the Pt-Pt axis of the complex.

methyl groups with the open coordination site at the metal atoms, followed by reorientation of the solvent molecules to specifically coordinate Ir atoms with the more electronegative cyano endings. In both complexes a metal-metal bond is formed after photoexcitation, thus effectively shifting electronic density from the outer side of the planar faces of the molecules to the inside (see the Supporting Information for an analysis of the electronic density difference between the $\mathrm{S}_{0}$ and $\mathrm{S}_{1}$ states of PtPOP as obtained from the vacuum calculations performed in this work). Although different solvents are involved in the two cases, the different response of coordinating solvent molecules can be rationalized in terms of different contributions of atomic orbitals localized on ligand atoms in the formation of the LUMO. For PtPOP, the LUMO has a largely predominant $\mathrm{p}_{z}$ character; as a consequence, in the excited state, a considerable portion of the electronic density still localizes in outward position with respect to the planar $\mathrm{PtP}_{4}$ faces (see Figure $\mathrm{S} 2$ in the Supporting Information). 
This, in turn, permits the Pt atoms of the complex to retain their ability to coordinate the more electropositive part of the solvent and it is probably connected to the previously mentioned excited-state reactivity towards $\mathrm{H}$ atom donors. For $\left[\operatorname{Ir}_{2}(\operatorname{dimen})_{4}\right]^{2+}$, on the other hand, previous DFT calculations ${ }^{7}$ have highlighted a substantial involvement of $\pi_{z}^{*}(\mathrm{C} \equiv \mathrm{N})$ orbitals in the formation of the LUMO, shifting more electron density from the outer sides of the molecule and making the excited state a stronger Lewis acid.

\section{Vibrational dynamics in vacuum}

We have performed two different types of $\triangle \mathrm{SCF} B O M D$ simulations of $\mathrm{S}_{1} \mathrm{PtPOP}$ in vacuum. In the first simulation, a single $\mathrm{S}_{1}$ trajectory was started from a structure geometry-optimized in vacuum in the $\mathrm{S}_{1}$ state with respect to all DOF except for the Pt-Pt distance, which was set at the value of the ground-state optimized structure $(3.005 \AA)$. At the beginning of the simulation all atomic momenta were equal to 0 . The trajectory was then propagated for $16 \mathrm{ps}$ with time step of $1 \mathrm{fs}$. While this choice of initial conditions is far from being representative of the state created by excitation with an ultrashort laser, it nevertheless provides a useful means for more easily identifying vibrational modes of the molecule that couple more strongly to the Pt-Pt stretching mode, since at the beginning of the dynamics almost all excess potential energy will be concentrated in this mode. In a second vacuum $\triangle \mathrm{SCF} B O M D$ simulation, we have propagated a $\mathrm{S}_{1}$ trajectory starting from the optimized geometry of the ground state. This second choice of initial conditions corresponds to a CW (infinitely long) pump pulse. In which case, the excitation window of equation (17) is a delta function and only one trajectory is propagated (this is the Bersohn-Zewail (BZ) model, see

for example ${ }^{87}$ ). This simulation was aimed at producing a picture of the dynamics that is closer to the events that take place in an ultrafast pump-probe experiment than the one that emerges from the first simulation. Total propagation time and time step were the same as those of the $\triangle \mathrm{SCF}$ BOMD run started from a relaxed $\mathrm{S}_{1}$ geometry with the Pt-Pt distance of the ground state. 
In the first vacuum simulation, the Pt-Pt stretching mode takes up alone almost all excess vibrational energy at the beginning of the dynamics. This is most apparent from Figure 5, which reports at different interval of times during the simulation the percent fraction of average total energy (kinetic plus potential) for the four modes that were found to have the largest average kinetic energy over the entire simulation time and for the sum of the rest. The average total energy for each mode was calculated from the virial theorem as twice the

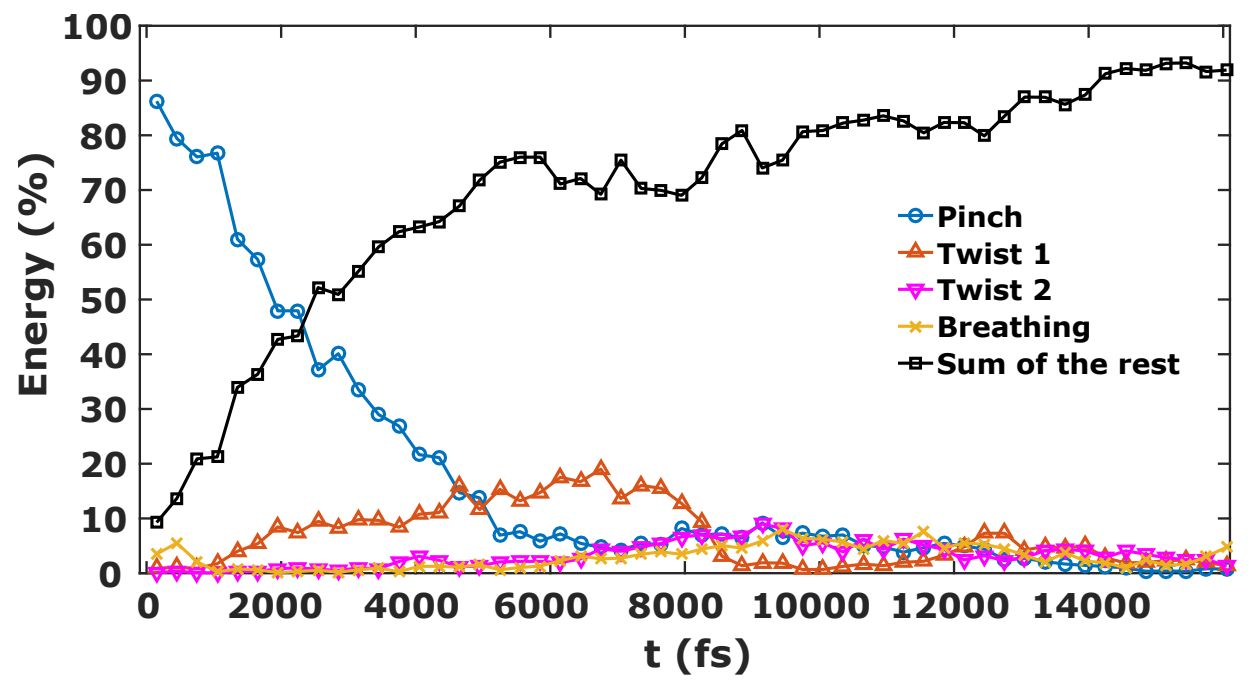

Figure 5: Time evolution of the total energies (kinetic plus potential) of selected vibrational modes and the sum of the rest as obtained from a generalized normal mode analysis of a gas-phase $\mathrm{S}_{1}$ trajectory started from a PtPOP structure with the Pt-Pt distance of the ground state and all other DOF relaxed in the $\mathrm{S}_{1}$ state. In total, the modes extracted from the vibrational analysis after removing the translations and overall rotations, and taking into account the constraints enforced on the positions of the hydrogen atoms during the dynamics, were 92. The total mode energies were averaged over time intervals of 300 fs and expressed as a percentage of the total average vibrational energy. See Figure 6 for a depiction of the four selected modes

average of the kinetic energy over intervals of 300 fs. The four selected modes are depicted in terms of generalized normal mode displacement vectors in Figure 6. The figure also shows the Fourier transform (FT) of the autocorrelation function $C_{i}(t)$ of the mode velocities for each mode. These were calculated from:

$$
C_{i}(t)=\left\langle\dot{Q}_{i}(0) \dot{Q}_{i}(t)\right\rangle
$$




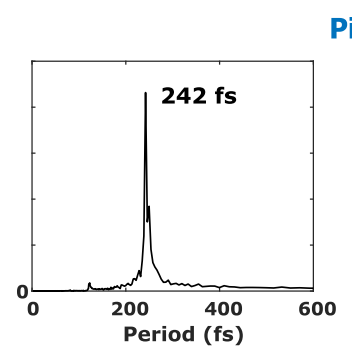

Pinch
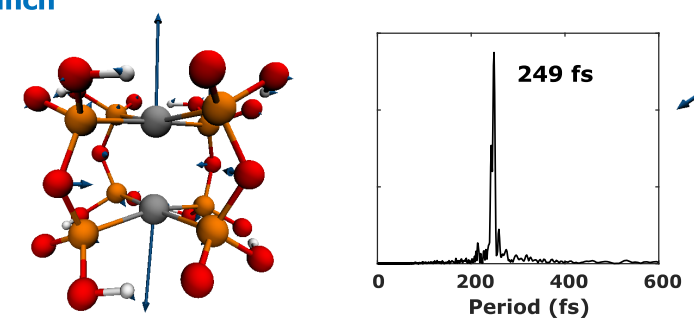

Twist 1
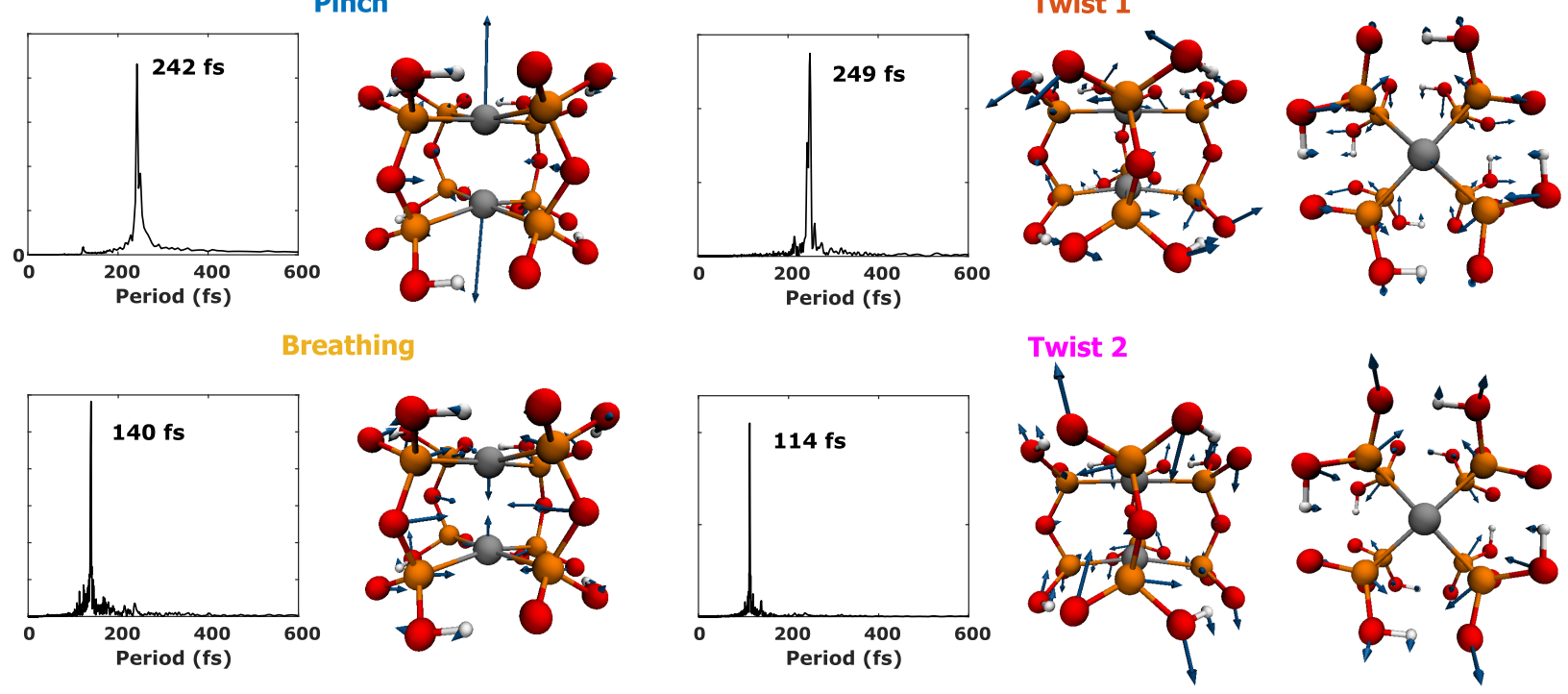

Figure 6: The four main generalized normal modes involved in the gas-phase $\mathrm{S}_{1}$ dynamics of PtPOP, and the FTs of their velocity autocorrelation functions. The position of the maximum of the FT of a mode gives the characteristic frequency of that mode.

The positions of the FT peaks represent the characteristic vibrational frequencies of the modes. The mode with character of Pt-Pt stretching is indicated as "pinch". Initially in the dynamics, this mode takes up to almost $90 \%$ of the total vibrational energy. After around $6 \mathrm{ps}$, the portion of energy shared by the pinching mode has decreased by around $95 \%$ of the initial value. Of this, $\sim 80 \%$ has flowed into 87 modes, which seem to be activated simultaneously and at the same rate, with none of them showing particular preference for overtaking the excess Pt-Pt vibrational energy; while around $20 \%$ has been transferred to a single mode with main character of ligand twist (twist 1). Thereafter, a significant portion of the energy flow is directed towards the other two remaining modes (twist 2 and breathing), which, thus, seem to be activated rather sequentially after the activation of twist 1 . The interplay between the four main modes identified in the vibrational analysis manifests itself in the evolution of the respective kinetic energies, as illustrated in Figure 7. In particular, the strong coupling between the pinching mode and twist 1 is evident from the fact that while the energy flow into twist 1 is at a maximum, around 6-7 ps, the kinetic energy of the pinch has reached a minimum, and after that has a small increase at the expense of 


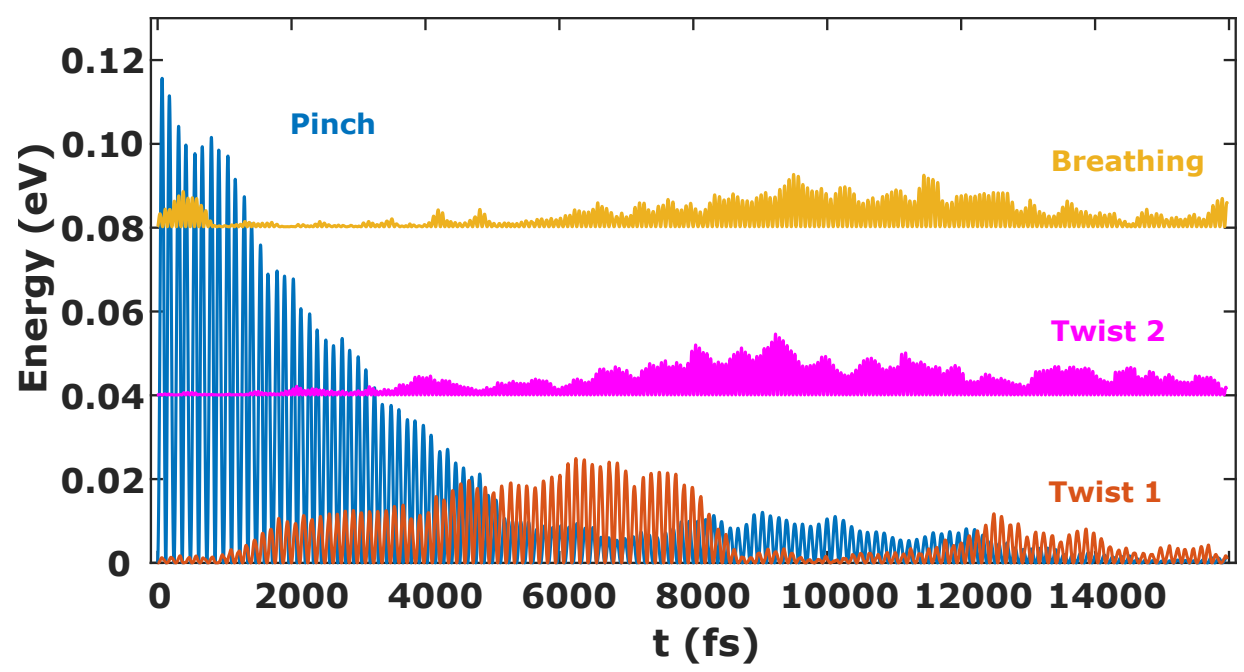

Figure 7: Instantaneous kinetic energy of the four main modes of a generalized normal mode analysis of PtPOP along a vacuum trajectory in $\mathrm{S}_{1}$ where almost all excess vibrational energy is initially stored along the Pt-Pt stretching coordinate. The energies of the twist 2 and breathing modes are shifted upwards for better clarity. All modes are visualized with the help of displacement vectors in Figure 6.

the energy accumulated in twist 1 . The same is true at early times in the dynamics for the pinching and breathing modes. In fact, at times earlier than $1 \mathrm{ps}$, energy is seen to rapidly flow in and out of the breathing mode, matching a local minimum in the evolution of the kinetic energy of the pinch. To shed light on the origin of the couplings between these vibrational modes, an analysis in terms of their characteristic frequencies (see Figure 6) and main structural distortions involved is needed. The Pt-Pt pinching period of 242 fs is in satisfactory agreement with the $\sim 230$ fs value extracted from the vibrational progression of the low-temperature $\mathrm{S}_{0} \rightarrow \mathrm{S}_{1}$ absorption band of crystal $\left(\mathrm{n}-\mathrm{Bu}{ }_{4} \mathrm{~N}\right)_{4}[\mathrm{PtPOP}],{ }^{7,50}$ already mentioned before. Interestingly, the breathing mode having also partial character of Pt-Pt stretching, thus explaining why these two modes seem to be coupled despite the breathing mode has a considerably higher frequency. Regarding the latter, the period of 140 fs obtained from the maximum of the FT of this mode is in very good agreement with the experimental $232 \mathrm{~cm}^{-1}$ peak (144 fs period) of the Raman spectrum of PtPOP in the ground state, ${ }^{100}$ which was assigned to a symmetric $\mathrm{Pt}_{2} \mathrm{P}_{8}$ stretching mode by Gellene and Roundhill ${ }^{74}$ on the basis of a DFT vibrational analysis. Lastly, twist 1 and twist 2 are antisymmetric twistings of the 
ligands, where pairs of opposite ligands twist in clockwise and counterclockwise directions, resulting in variations of the dihedral $\angle \mathrm{P}-\mathrm{Pt}-\mathrm{Pt}_{-} \mathrm{P}^{\prime}$ and in-plane $\angle \mathrm{P}-\mathrm{Pt}-\mathrm{P}$ angles. The strong coupling between the pinching and one of these twisting modes (twist 1) is readily explained by the fact that they share almost the same frequency. Indeed, the period of the twisting mode is found to be only $\sim 8$ fs longer than that of the pinch. A vibrational analysis carried out using the Gaussian09 program on the ground-state molecule optimized in vacuum with BLYP identified an analogous normal mode with frequency close to the one of the Pt-Pt stretching mode. Furthermore, the calculated gradients of the dipole moment and polarizability of PtPOP along this mode are very close to 0, revealing that it is neither IR or Raman active, thus explaining why it has never been observed experimentally (unfortunately Gellene and Roundhill ${ }^{74}$ have decided to report only DFT-calculated frequencies that could be compared to experimentally determined IR or Raman transitions, thus a comparison with their vibrational analysis cannot be made for this mode). Plots of the evolution of the structural parameters that are mostly involved in the dynamics of the selected modes, together with their FTs, are shown in Figure 8. The frequencies of the fluctuations of reported atomic displacements and angles, and the time evolution of their amplitudes correlate very well with the evolution of the mode kinetic energies shown in Figure 7, thus further validating the results of the generalized normal mode analysis. We note that it would be more difficult to infer the intramolecular energy flow from the local mode picture provided by the evolution of the amplitudes of oscillations of single structural parameters, when a priori knowledge of the coupled nuclear motion in the dynamics is lacking. This is clear, for example, from the plot of the evolution of the average P-P distance between $\mathrm{P}$ atoms belonging to the same $\mathrm{PtP}_{4}$ group and to opposite ligands, which features two superimposed oscillations with different frequencies (indeed, the P-P distances change both in the breathing and pinching modes); as a consequence, the correlation between the pinching and breathing modes before 1 ps, which shows up clearly in the evolution of the kinetic energies, is lost, thus highlighting the advantages offered by a decomposition of the kinetic energy in generalized normal mode 

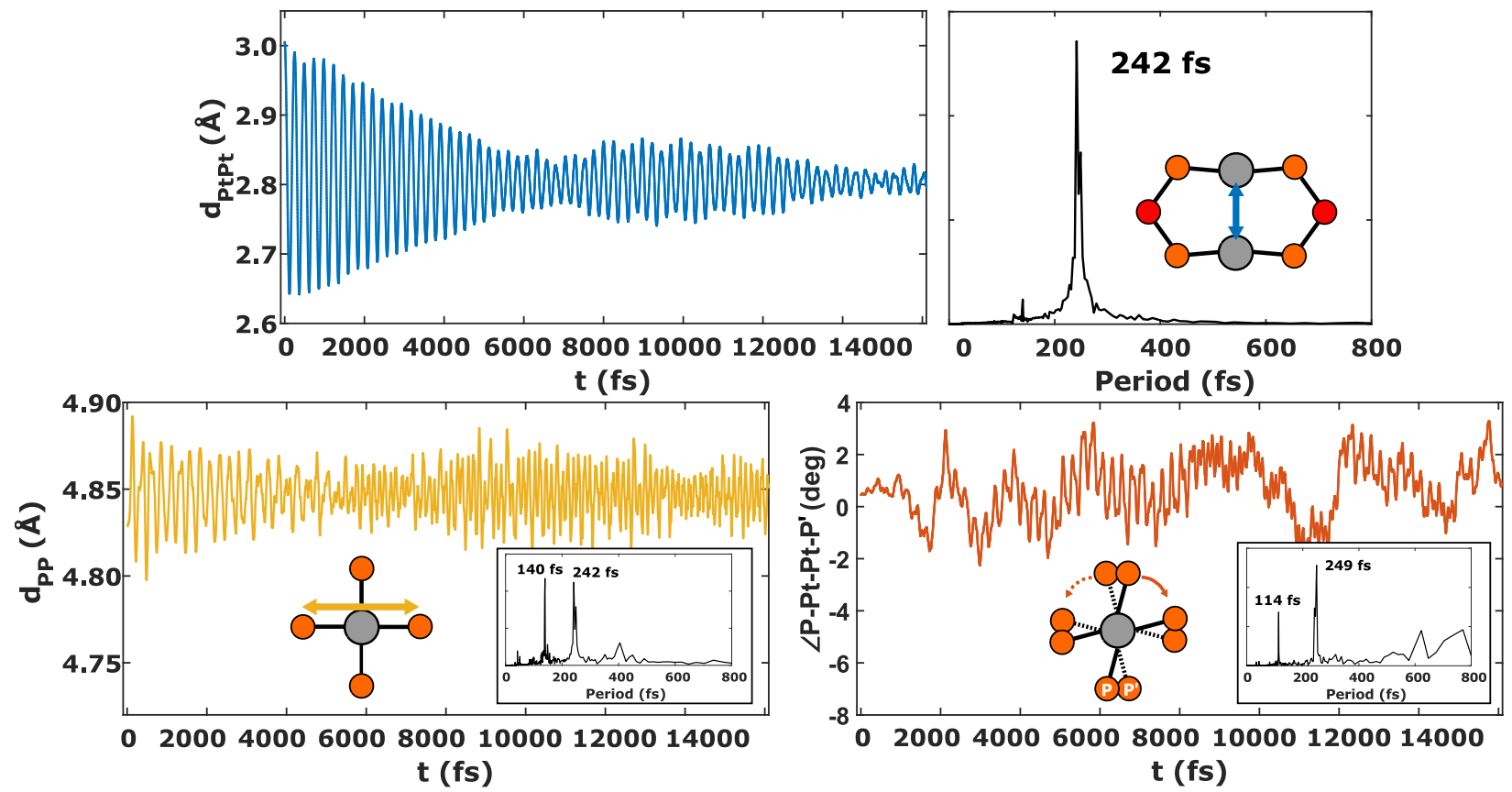

Figure 8: Evolution of the main geometry parameters of PtPOP involved in the $\Delta \mathrm{SCF}$ BOMD vacuum simulation started from a relaxed $S_{1}$ structure with the Pt-Pt distance of the ground-state optimized geometry, and their FT. Changes in the reported parameters and their frequencies correlate with variations in the kinetic energy of the normal modes presented in Figure 7. (Top) Evolution of the Pt-Pt distance. (Bottom, left) Instantaneous average P-P distance between $\mathrm{P}$ atoms belonging to opposite ligands. Since P-P symmetric vibrations take part in both the breathing and pinching modes, fluctuations in this parameter reflect the frequencies of both modes. (Bottom, right) Variation of the mean of the $\angle \mathrm{P}-\mathrm{Pt}$ $\mathrm{Pt}^{-\mathrm{P}^{\prime}}$ dihedral angles involving ligands that undergo simultaneous clockwise torsion in the dynamics of the twist 1 and twist 2 modes.

contributions as performed in this work. Overall, we can conclude that the dynamics is dominated by the pinching mode, which is initially activated, and those few modes that are more strongly coupled to it. In the case of the breathing mode, the coupling can be explained by the partial character of Pt-Pt stretching that this mode presents; while in the case of the twist 1 mode, the coupling derives from its period being very close to that of the pinching mode. Finally, the twist 2 mode is involved in the dynamics because it has a very similar character to, and hence is coupled to, the twist 1 mode.

In the second $\triangle \mathrm{SCF} B O M D$ vacuum simulation, started from the ground-state optimized geometry of the complex, the portion of total energy stored initially in the pinching mode was found to be much smaller than in the first simulation, being equal to only $\sim 30 \%$ (see 
Figure S11 in the Supporting Information). Notably, an almost equal portion of energy is shared by a mode that was not activated in the first simulation. This mode is depicted in Figure 9 (right), where also the FT of the autocorrelation of mode velocities is reported. It
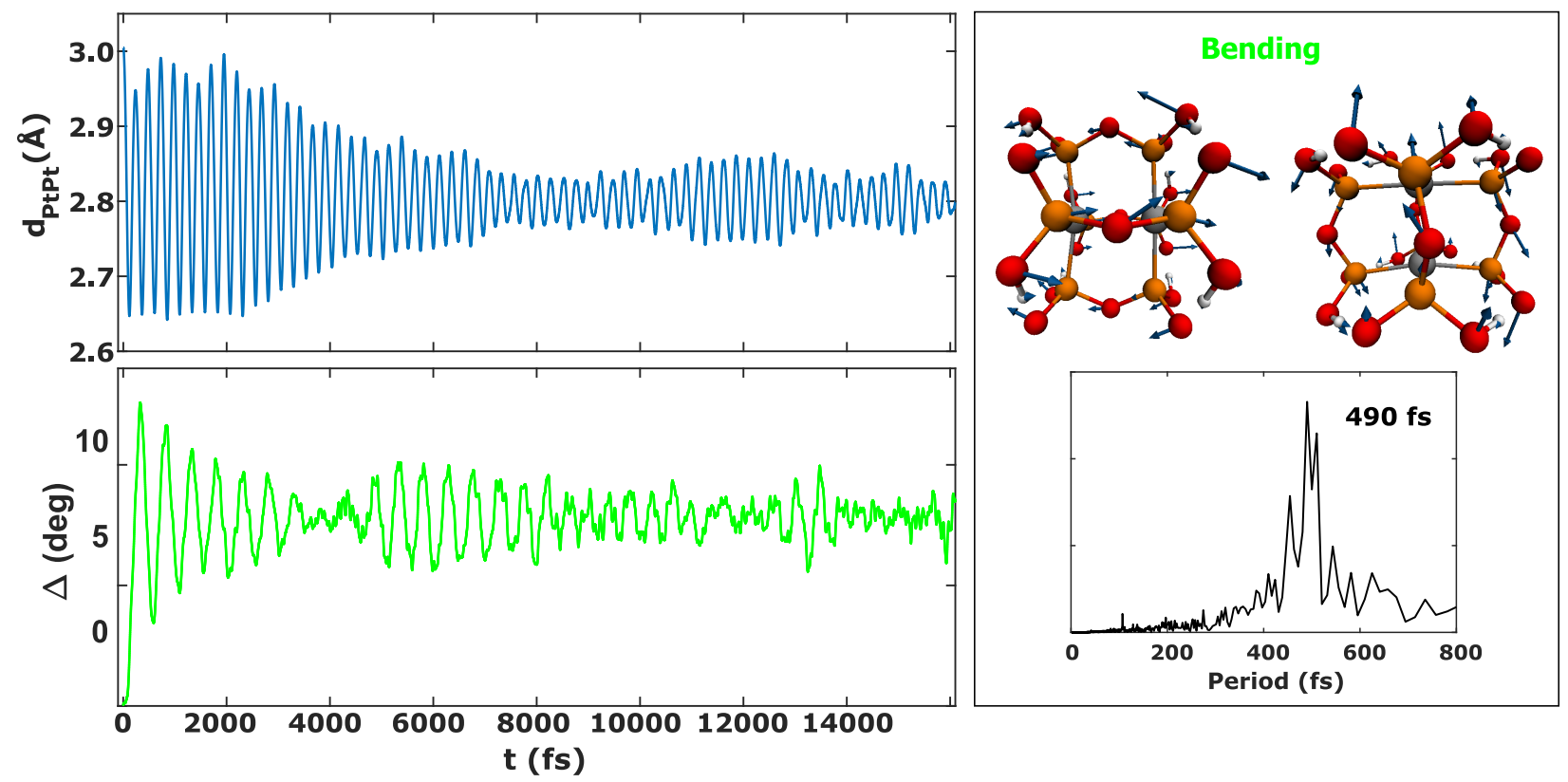

Figure 9: Evolution of the vacuum $\mathrm{S}_{1}$ trajectory started from the optimized gas-phase $\mathrm{S}_{0}$ geometry of PtPOP along the coordinates that, at the beginning, share the largest portion of vibrational energy of the molecule. (Top, left) Variation of the Pt-Pt distance. (Bottom, left) Evolution of the pseudorotation coordinate $\Delta$ defined in Figure 2. (Right) Visualization of the mode that corresponds to motion along $\Delta$ and is activated at the beginning of the dynamics together with the pinching mode.

is characterized by a bending of the ligands corresponding to nuclear motion in the well of the potential energy landscape along the pseudorotation coordinate $\Delta$, as defined in Figure 2. This is further confirmed by the evolution of $\Delta$ during the dynamics (Figure 9 (bottom left)), which shows a first rapid increase, followed by oscillations with a period of $\sim 490 \mathrm{fs}$ around a value of about $7^{\circ}$, consistent with the shape and the minimum of the potential shown in Figure 2. The only experimental indication of the existence of a vibrational mode with a lower frequency than the metal-metal stretching in PtPOP is given by the presence of a $\sim 40 \mathrm{~cm}^{-1}$ sideband on the Pt-Pt vibronic progression of low-temperature absorption and emission spectra of single crystals of $\mathrm{Ba}_{2}[\mathrm{PtPOP}],{ }^{51}$ which was attributed to a ligand deformation mode, but was never further characterized. According to the results of our 
simulations we assign the observed mode to a bending of the ligands in a $D_{2 d}$ geometry (of the $\mathrm{Pt}_{2} \mathrm{P}_{8}$ core), thus reaffirming our conclusion that $\mathrm{PtPOP}$ in the excited state does not retain a $C_{4 h}$ symmetry. In Figure 10 we report the evolution of the instantaneous kinetic energy along the modes with the largest average kinetic energy over the entire MD simulation time. These include the pinching and bending modes, the breathing and twist 1 modes, identified also previously, and a new twisting mode (twist 3). The behaviour of the twist 3 mode is similar to the twist 2 mode observed for the first vacuum $\triangle \mathrm{SCF}$ BOMD simulation, in that it is activated later in the dynamics, after about 6 ps, but has a slightly different period ( $\sim 107 \mathrm{fs}$ ) and character of the torsional motion (see Figure S12 in the Supporting Information). The energy along the bending mode is seen to decrease rapidly in the first

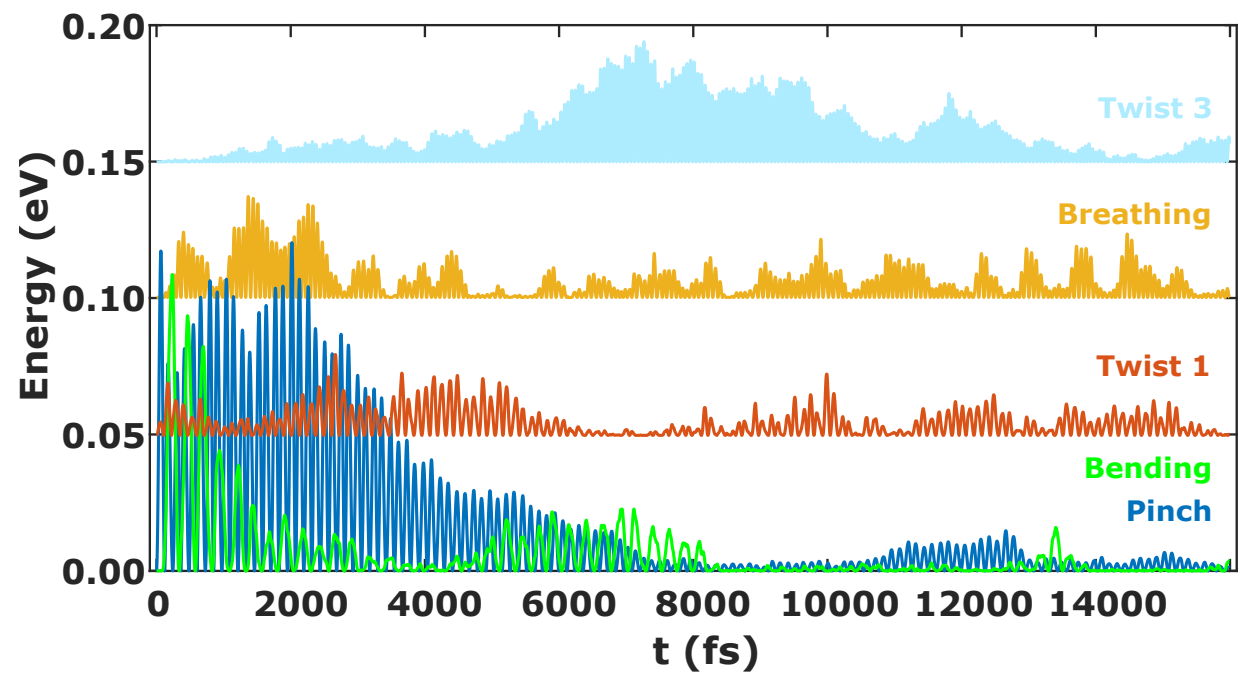

Figure 10: Evolution of the instantaneous kinetic energy along the vibrational modes of $\mathrm{PtPOP}$ that undergo the largest displacements during a vacuum BOMD propagation in $\mathrm{S}_{1}$ started from the ground-state optimized geometry of the complex.

$\sim 3$ ps. The pinching mode, instead, has, at $\sim 3 \mathrm{ps,} \mathrm{around} \mathrm{the} \mathrm{same} \mathrm{kinetic} \mathrm{energy} \mathrm{it} \mathrm{had}$ at the beginning; hence, it is reasonable to assume that a considerable portion of the excess energy of the bending flows to the pinch. Besides, we observe how, in this second type of simulation, the coupling between the pinching and the breathing and twist 1 modes seems to be accentuated. This is apparent from the multiple local dips that characterize the evolution of the kinetic energy of the pinch in the first $\sim 3 \mathrm{ps}$, which are accompanied by variations 
of the same magnitude but opposite sign in the energy of the breathing and twist 1 modes. What is new with respect to the first simulation, in which all coordinates except the Pt-Pt distance were relaxed before running the dynamics, is that a non-negligible portion of the total kinetic energy of the molecule is also stored in the breathing and twisting modes from the very beginning of the dynamics. Therefore, we conclude that initial activation following the gradients of the excited-state potential after excitation can induce a stronger coupling of the ligand deformation modes with the Pt-Pt vibrations along the dynamics.

\section{Solution dynamics}

Figure 11 shows a schematic of the excitation process simulated in aqueous solution, together with plots of the evolution of the distribution of Pt-Pt distances as obtained from the ensemble of out-of-equilibrium $\mathrm{S}_{1} \Delta \mathrm{SCF}-\mathrm{QM} / \mathrm{MM}$ trajectories. The excited population exhibits coherent Pt-Pt oscillations around the equilibrium distance of $2.79 \AA$ with a period of $\sim 230$ fs that persist up until around 2 ps. The value of the vibrational period is found in remarkably good agreement with the experimental period of $224.5 \pm 0.1$ fs measured by van der Veen et al. ${ }^{14}$ in a femtosecond transient absorption experiment in water. So far we have shown that the Pt-Pt distance is not the only coordinate to undergo large changes from ground to excited state, but the Pt-Pt contraction is accompanied by a bending of the ligands, quantified by an increase of the parameter $\Delta$ by $\sim 5^{\circ}$. Therefore, we have examined the possibility that the ensemble of excited molecules displays coherent oscillations also along the coordinate $\Delta$ by plotting the evolution of the distribution of angle differences $\Delta$ (Figure 12). As apparent from Figure 12, no coherent oscillations are observed for the bending motion, but rather the trajectories along this coordinate exhibit the behaviour of overdamped oscillators, reaching the equilibrium value gradually over a time of $\sim 2.5 \mathrm{ps}$. This finding is consistent with the lack of oscillating signatures different from the Pt-Pt stretching vibrations in time-resolved measurements in solution. ${ }^{14}$ Focusing back on the Pt-Pt oscillations, we have investigated the causes of the decoherence by quantifying the coherence decay $\left(\tau_{c}\right)$ and vibrational cooling 

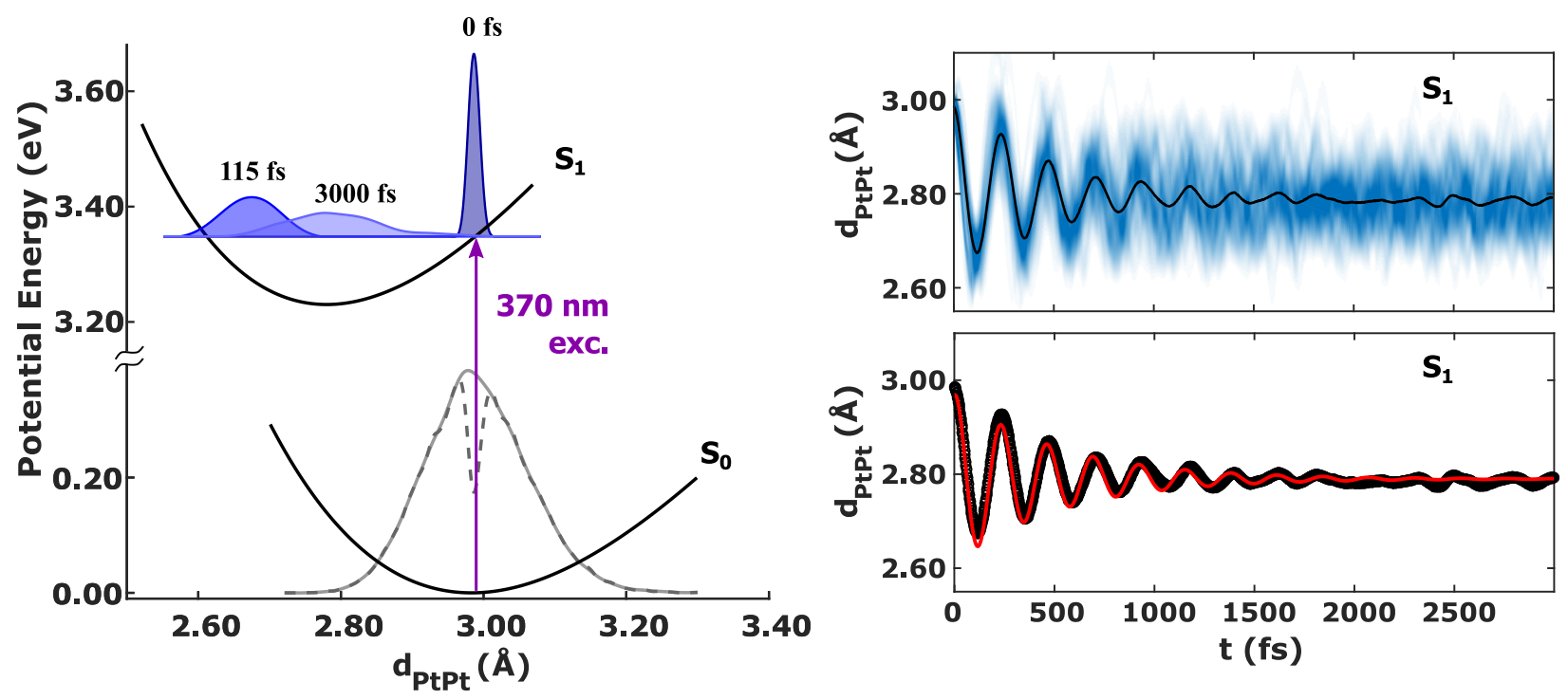

Figure 11: (Left) Schematics of the simulated $\mathrm{S}_{0} \rightarrow \mathrm{S}_{1}$ photoexcitation of PtPOP in aqueous solution. The $\mathrm{S}_{0}$ and $\mathrm{S}_{1}$ potentials used within the $\mathrm{SF}$ approximation of the excitation (see equations (16) and (17)) are Morse-potential fits to the PMF obtained as explained in the Computational methods section, while the parameters of the laser pulse appearing in the expression of the excitation window $F\left(d_{\mathrm{PtPt}}\right)$, equation $(17)$, were chosen such to reproduce the experimental conditions of the transient absorption experiment of van der Veen et al. ${ }^{14}$ as close as possible. The continuous gray line is the equilibrium QM/MM distribution of $\mathrm{Pt}-\mathrm{Pt}$ distances in the ground state. The portion of molecules promoted to $\mathrm{S}_{1}$ according to equation (16) was subtracted from the equilibrium $S_{0}$ distribution to give the (unnormalized) distribution represented by the dashed line. The non-stationary distribution propagated in $\mathrm{S}_{1}$ by $\triangle \mathrm{SCF}-\mathrm{QM} / \mathrm{MM}$ BOMD (blue curves) is shown immediately after excitation, at its first classical turning point and at the end of the nonequilibrium dynamics. (Right, top) Density plot of the evolution of the ensemble of Pt-Pt distances obtained from propagation of $49 \Delta \mathrm{SCF}-\mathrm{QM} / \mathrm{MM}$ trajectories in $\mathrm{S}_{1}$ satisfying the initial conditions shown in the left panel. The superimposed black curve represent the instantaneous average Pt-Pt distance. (Right, bottom) Time dependence of the average Pt-Pt distance of the nonequilibrium $\mathrm{S}_{1}$ ensemble (black line) together with the best fit (red line) from equation (20). All displayed distributions were smoothed with a cubic smoothing spline.

$\left(\tau_{e}\right)$ times (see ${ }^{101}$ for an extensive discussion of these concepts) predicted by the simulations.

To obtain the simulation decoherence time, we have fitted the time-dependent average of Pt-Pt distances with a periodic monoexponentially decaying function of the form:

$$
f_{c}(t)=A \mathrm{e}^{-t / \tau_{c}} \cos \left(\frac{2 \pi}{T} t\right)+B
$$




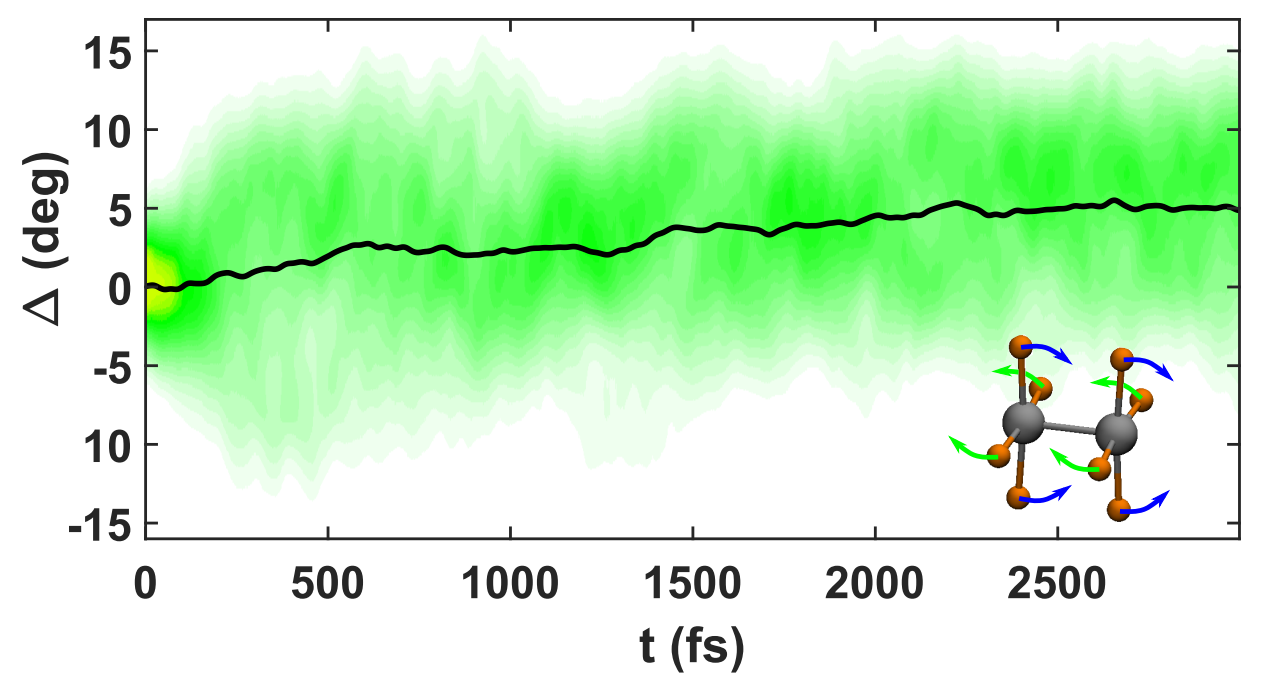

Figure 12: Density plot of the evolution of the distribution of angle differences $\Delta$ extracted from the ensemble of $\mathrm{S}_{1} \Delta \mathrm{SCF}-\mathrm{QM} / \mathrm{MM}$ trajectories of PtPOP in water. The black line is the mean $\Delta$ along the ensemble propagation. The distributions were smoothed with a cubic smoothing spline.

in which $T$ is the coherent oscillation period. Accordingly, the vibrational cooling time $\tau_{e}$ was computed by fitting the time-dependent kinetic energy of the pinching mode obtained from the generalized normal mode analysis, and shown in Figure 13, with the following function:

$$
f_{e}(t)=C \mathrm{e}^{-t / \tau_{e}} \cos ^{2}\left(\frac{2 \pi}{T} t+\frac{\pi}{2}\right)+D
$$

The best fits gave values of $\tau_{c}=520 \pm 14$ fs and $\tau_{e}=320 \pm 10$ fs. Relaxation of the vibrational energy competes, in solution, with pure dephasing processes, arising from elastic stochastic collisions with the solvent and phase changes along an anharmonic potential, in determining the final coherence time of the ensemble oscillations. We estimated the pure dephasing time $\left(\tau_{d}\right)$ by making use of the approximations underlying the optical Bloch equations. ${ }^{102}$ In the optical Bloch picture, the rate of decoherence is given, phenomenologically, by the sum of the rates of the two competing processes of vibrational cooling and pure dephasing:

$$
\frac{1}{\tau_{c}}=\frac{1}{2 \tau_{e}}+\frac{1}{\tau_{d}}
$$




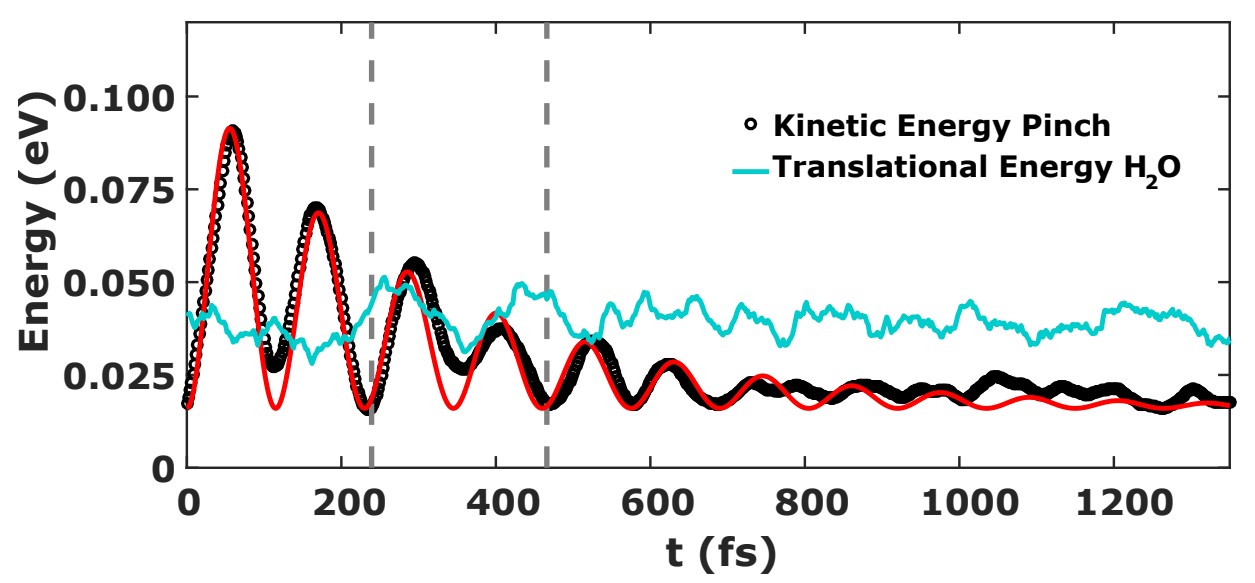

Figure 13: Time dependence of the kinetic energy of the pinching mode of PtPOP (black open circles) obtained from the vibrational analysis of the $\mathrm{S}_{1} \triangle \mathrm{SCF}-\mathrm{QM} / \mathrm{MM}$ BOMD simulations and averaged over all trajectories. The red line is the best fit of the function in equation (21) to the time dependence of the average pinching kinetic energy. Also shown (blue line) is the average translational kinetic energy of water molecules sampled by requiring that (i) at $t=0$ they are within the first peak of the Pt- $\mathrm{O}_{\text {solvent }} \mathrm{RDF}$ (see Figure 3 ) and (ii) at the end of the nonequilibrium propagation they have not left this coordination shell. Finally, the dashed vertical lines represent the times when the average Pt-Pt distance of the ensemble of non-stationary $\mathrm{S}_{1} \mathrm{PtPOP}$ molecules is at the first two outer turning points.

Using equation (22), a value of $2770 \mathrm{fs}$ is found for $\tau_{d}$. This means that the decoherence of the $\mathrm{Pt}-\mathrm{Pt}$ vibrations is essentially driven by energy dissipation along the Pt-Pt coordinate, while statistical effects are far less important. Experimentally, decoherence times of $\tau_{c}=1.76 \pm 0.8$ ps and $\tau_{c}=1.5 \pm 0.5$ ps were found from transient absorption and time-resolved fluorescence up-conversion measurements respectively. ${ }^{14}$ Furthermore, vibrational cooling was found to happen on time scales of $\tau_{c}=1.31 \pm 0.04 \mathrm{ps}$ (transient absorption) and $\tau_{c}=1.5 \pm 0.2 \mathrm{ps}$ (fluorescence up-conversion), i.e. simultaneously to coherence decay. Therefore, while the coherence decay is around three times faster in our simulations, they agree qualitatively with the experiments in the observation that the origin of the decoherence is mostly dynamical, i.e. a result of (dynamical) energy dissipation in the excited system, and not statistical in nature. This behaviour is a consequence of the compactness and rigidity of the scaffold of P-O-P ligands, the first providing screening of the Pt-Pt oscillator from (stochastic) interactions with solvent molecules, and the second offering a highly harmonic force constant for the pinching motion (the period of the oscillations in the average Pt-Pt distance from the 
simulations changes by only $\sim 18$ fs from the first to the last oscillation). As for the causes of the quantitative discrepancy between the coherence decay and vibrational relaxation times found for PtPOP from the present simulations with respect to the experimental values, we argue that this can be a consequence of different factors. One of the main cause derives from the calculations slightly overestimating the anharmonicity of the Pt-Pt motion. This is underpinned by the fact that the PMF computed from the equilibrated part of the $\mathrm{S}_{1} \Delta \mathrm{SCF}$ QM/MM trajectories, shown in Figure 11, is best fitted with a Morse potential, while in the transient absorption measurements performed by van der Veen et al. ${ }^{14}$ the period changes at most by $\sim 1.5$ fs in going from a 360 to a $380 \mathrm{~nm}$ excitation wavelength (as already mentioned, in our simulations the period changes by $\sim 18$ fs at the end of the coherent dynamics). Another possible cause of the discrepancy with the experimental values of coherence decay and vibrational relaxation times could be an overestimation of solvent-induced fluctuations in the energies of the KS orbitals in the $\triangle \mathrm{SCF}-\mathrm{QM} / \mathrm{MM}$ BOMD simulations. These fluctuations could favour temporarily other electronic states getting closer to S1, which, in turn, would lead to a faster dephasing of the QM/MM trajectories

The mechanism of coherence decay in PtPOP is different from what was proposed for the $\left[\operatorname{Ir}_{2}(\text { dimen })_{4}\right]^{2+}$ complex ${ }^{29}$ mentioned previously. The main factor causing decoherence in $\left[\operatorname{Ir}_{2}(\text { dimen })_{4}\right]^{2+}$ is, in fact, statistical, ascribable to the flexibility of the dimen ligands that impart higher anharmonicity to the potential energy surface and a broader width to the distribution of configurations of ground-state molecules that can be excited. ${ }^{7,29}$ Even more insightful is, perhaps, a comparison with the behaviour observed for $\mathrm{I}_{2}$ undergoing geminate recombination after photoexcitation in different environments. When the reaction was followed in solvents like $\mathrm{CCl}_{4}$ or cyclohexane, vibrational relaxation was found to occur without coherent oscillations. ${ }^{103}$ The behaviour of PtPOP is, instead, much more similar to that of $\mathrm{I}_{2}$ in solid krypton, where stochastic collisions with solvent molecules leading to dephasing in solution are absent and the system is allowed to dissipate energy while retaining the vibrational phase. ${ }^{102,104}$ In all cases, the rigidity of the environment surrounding the 
oscillators is found to play an important role in determining whether vibrational coherence survives during the energy relaxation process or not.

Having established that vibrational cooling drives the coherence decay of the ensemble of Pt-Pt oscillators, the natural question that arises at this point is: what are the paths of energy dissipation from the Pt-Pt coordinate? To provide an answer to this question, we have first investigated the hypothesis advanced by van der Veen et al. ${ }^{14}$ that the main channel of energy dissipation involves transient orientationally specific interactions of the Pt atoms with water molecules coordinated at the open axial site. To do so, we have calculated the average translational and rotational energies of water molecules selected from the first solvent coordination shell around the Pt atoms defined by the first peak of the Pt- $\mathrm{O}_{\text {solvent }} \mathrm{RDF}$, as indicated in Figure 3. The average translational energy is plotted as a function of time in Figure 13 together with the average kinetic energy along the pinching mode. The average rotational energy extracted from the trajectories did not display any particular displacement from its equilibrium thermal value, and, thus, it's not reported here. Early in the dynamics, the average translational energy of the coordinating water molecules experiences small positive fluctuations from its thermal equilibrium value. These fluctuations happen at around 250 and 450 fs, i.e. at the first and second outer turning points of the average Pt-Pt distance. This uptake of energy by the solvent, however, represents only a small fraction of the loss of energy from the pinching mode, and, certainly, cannot explain the steady decrease happening already during the first Pt-Pt oscillation period. In other words, the water molecules are more "spectators" of the Pt-Pt dynamics, rather than active participants in the relaxation process. This is further substantiated by the time evolution of the first peak of the Pt- $\mathrm{O}_{\text {solvent }}$ RDF presented in Figure 14. The oscillations that appear until around 500 fs mirror the Pt-Pt oscillations of the excited-state ensemble of PtPOP molecules, thus implying that the solvent molecules are relatively static during this part of the dynamics. After that, the Pt-Pt distribution has almost reached an equilibrium, and the solvent molecules rearrange to the new solute configuration, as evident from the inset 


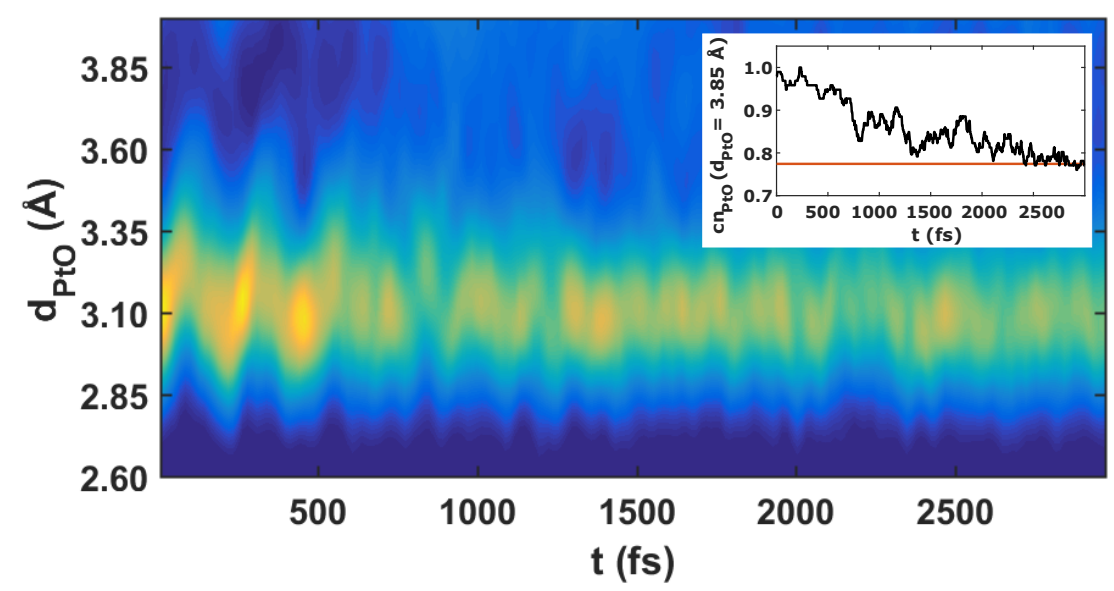

Figure 14: Density plot of the time evolution of the first peak of the Pt- $\mathrm{O}_{\text {solvent }} \mathrm{RDF}$ obtained from the $\mathrm{S}_{1} \triangle \mathrm{SCF}-\mathrm{QM} / \mathrm{MM}$ trajectories of PtPOP in water during the first 3 ps of dynamics. The inset shows the time dependence (black curve) of the cumulative $\mathrm{Pt}-\mathrm{O}_{\text {solvent }}$ coordination number at $d_{\mathrm{PtO}}=3.85 \AA$, representing the instantaneous average number of water molecules found within the first solvent coordination shell of the Pt atoms, together with the value (red line) obtained from the equilibrated part of the $\triangle \mathrm{SCF}-\mathrm{QM} / \mathrm{MM}$ trajectories.

of Figure 14, which reports the time evolution of the Pt- $\mathrm{O}_{\text {solvent }}$ cumulative coordination number at the first minimum of the $\operatorname{RDF}\left(d_{\mathrm{PtO}}=3.85 \AA\right)$. Since only water molecules coordinating to the $\mathrm{Pt}$ atoms at the free axial sites are eligible to accept energy directly from the Pt-Pt pinching, the simulations seem to exclude direct solute-solvent interactions as the main source of energy loss. Therefore, other intramolecular vibrational modes have to mediate dissipation of the excess energy along the Pt-Pt coordinate to the solvent owing to anharmonic couplings with the pinching mode. A second indication that this is indeed the case is given by Figure 15 (top), where the total vibrational energy of PtPOP averaged over time intervals of $100 \mathrm{fs}$ is plotted. An exponential fit to the evolution of the total vibrational energy, also shown in Figure 15 (top), gives a time constant of $600 \pm 200$ fs (uncertainty estimated from the $95 \%$ confidence bound of the fitting parameter) for the decay before reaching equilibrium. Hence, the total vibrational energy is dissipated almost twice as slow as the vibrational cooling along the Pt-Pt coordinate. It is reasonable to expect that the energy put into vibrational modes involving ligand atoms in the excitation process is dissipated faster to the solvent than the Pt-Pt excess energy since the ligands 

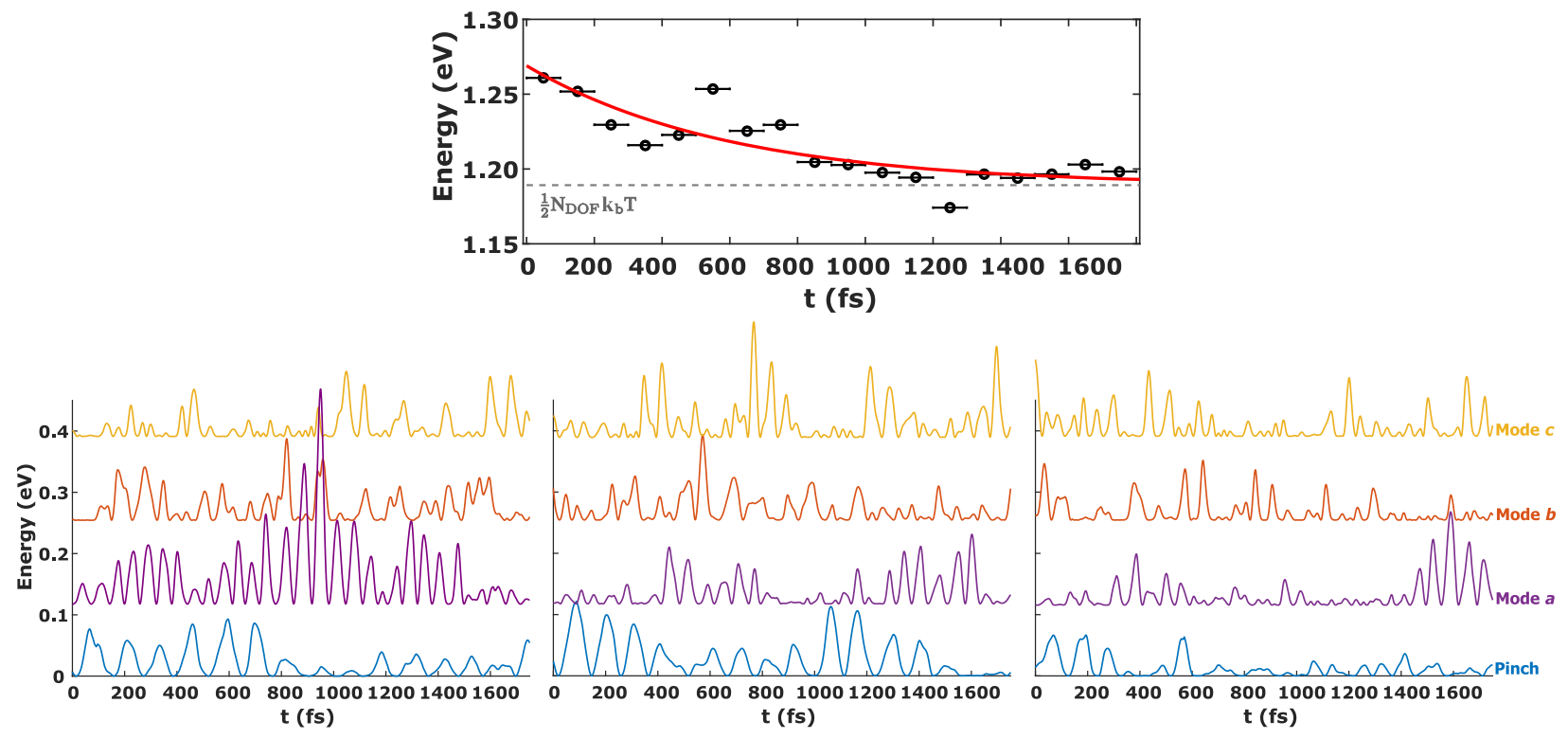

Figure 15: (Top) Evolution of the ensemble average total vibrational energy of PtPOP (black circles) obtained as sum of the kinetic energies of the individual generalized normal modes, according to equation (11), from the vibrational analysis of the $\mathrm{S}_{1} \Delta \mathrm{SCF}-\mathrm{QM} / \mathrm{MM}$ trajectories. The average ensemble total energy was further averaged over time intervals of 100 fs as indicated by the horizontal black lines. The red line is an exponential fit to the data points, while the horizontal dashed line represents the theoretical value of vibrational energy of an ensemble of molecules with the number of DOF of PtPOP in the simulations in equilibrium at $300 \mathrm{~K}$. (Bottom) Plots of the time dependence of the kinetic energy along selected vibrational modes of PtPOP for three representative $\triangle \mathrm{SCF}-\mathrm{QM} / \mathrm{MM}$ trajectories in $\mathrm{S}_{1}$. The kinetic energies of modes $a, b$ and $c$ are vertically shifted for clarity of presentation.

are more exposed to direct interactions with the solvent. It follows that the above result can be interpreted as an indication that the energy initially stored in the Pt-Pt coordinate might survive in the PtPOP molecule for longer than the simulated vibrational cooling time for the ensemble of Pt-Pt oscillators. As a last, more stringent, test of this mechanistic hypothesis we have plotted in Figure 15 (bottom) the evolution of the kinetic energy for the pinching mode together with three other relevant vibrational modes, as obtained from the generalized normal mode analysis, along three representative trajectories. The modes labelled mode $b$ and mode $c$ were found to have similar frequencies and large overlaps with respectively the twist 1 and breathing modes obtained from the gas-phase trajectories, and shown previously to be coupled to the pinching mode. However, they cannot be characterized fully as a twisting and a breathing mode, since they exhibit also character of other types 
of vibrations, most significantly Pt-P stretching (a representation of the modes in terms of displacement vectors is given in Figure S13 in the Supporting Information). Mode $a$ does not overlap significantly with any of the main modes coupled in vacuum with the Pt-Pt pinch. It has mixed character of Pt-P stretching and ligand twist, with an autocorrelation function of mode velocities (see Figure S13) centered around 120 fs. This period is not far from the position of the peak (138 fs) in the IR spectrum of PtPOP assigned to P-Pt-P stretching by Gellene and Roundhill. ${ }^{74}$ Notably, the evolution of the kinetic energies along these ligand deformation modes is seen from Figure 15 (bottom) to be strongly anticorrelated with the evolution of the kinetic energy of the pinching mode, since drops in the latter are always mirrored by increments of the former and vice versa. P-O and P-OH groups in the molecule experience large nuclear motion along the three ligand vibrational modes. Since these groups are likely involved in hydrogen bonding with the water molecules during the dynamics, the modes are expected to efficiently funnel excess energy to the solvent.

Overall, the simulations carry clear signs that dissipation of the Pt-Pt energy to the solvent, which drives the decoherence of the Pt-Pt oscillations, occurs mainly indirectly through IVR to modes characterized by motion of the O-P-OH moieties. The result seems to confirm the hypothesis recently put forward by Monni et al. ${ }^{10}$ mentioned in the introduction, that anharmonic couplings between internal modes are the main source of decoherence of the Pt-Pt vibrations in photoexcited PtPOP. Since vibrational cooling along the pinching mode is found from the simulations to be much faster in solvent compared to vacuum, we can deduce that the role of the solvent is actually to facilitate anharmonic couplings between the modes, making IVR more efficient. Experimentally, van der Veen et al. ${ }^{14}$ found a dependence of vibrational cooling to the solvent, which was interpreted as a signature of direct energy transfer from the Pt-Pt coordinate to the solvent. This interpretation, however, neglects the fact that different solvents can affect the strength of the anharmonic couplings between internal modes differently, thus changing the rates of IVR. Once again, this is in contrast to what was found for $\left[\operatorname{Ir}_{2}(\text { dimen })_{4}\right]^{2+}$ in acetonitrile, where the solvent prolongs coherence of 
the metal-metal oscillations by making, in some cases, IVR less likely than in vacuum. ${ }^{29}$

\section{Conclusions}

In this work, we have shed light on the structural and solution dynamics of the first excited state of the model photocatalyst PtPOP by performing PESs calculations and nonequilibrium vacuum and $\mathrm{QM} / \mathrm{MM}$ BOMD simulations. A modified version of $\triangle \mathrm{SCF}$ was used to

obtain the electronic structure of $\mathrm{S}_{1}$. The approach relies on a Gaussian smearing of the discrete $\triangle \mathrm{SCF}$ constraints on the orbital occupation numbers to readily converge the electronic density of the complex during the dynamics, and was implemented in the GPAW DFT code. The computational expediency of the method was exploited to collect large amount of statistics in order to unambiguously assess the validity of the simulations against known experimental data.

We reported DFT-calculated PESs along the Pt-Pt coordinate for both the first $\mathrm{d} \sigma^{*} \rightarrow \mathrm{p} \sigma$ singlet and triplet excited states, for the first time providing computational evidence that they have approximately the same shape and position with respect to the ground-state gasphase equilibrium geometry. From a structural point of view, we also found that in the excited state $\mathrm{PtP}_{4}$ groups are distorted towards a local quasi-trigonal bipyramidal geometry, thus lowering the $\mathrm{Pt}_{2} \mathrm{P}_{8}$ core symmetry to $D_{2 d}$; an aspect that had passed unnnoticed from previous DFT calculations but which could play a decisive role in determining the trends observed in the ISC rates of PtPOP and its derivatives in solution. ${ }^{7,58,60}$ This distortion is associated to the presence of a low-frequency mode in the molecule with character of bending of the ligands, which in the gas-phase $\triangle \mathrm{SCF}$ BOMD simulations manifests itself with oscillations with a period of $\sim 490 \mathrm{fs}\left(68 \mathrm{~cm}^{-1}\right)$. Based on this, we tentatively assigned the $40 \mathrm{~cm}^{-1}$ sideband superimposed to the Pt-Pt stretch progression in low-temperature absorption spectra of PtPOP in crystals, ${ }^{51}$ which had so far remained uncharacterized, to this ligand distortion mode. 
As for the ultrafast dynamics following photoexcitation to $\mathrm{S}_{1}$ in water, we have established the paths of energy flow from the Pt-Pt stretching vibrations to the solvent. Water molecules strongly coordinated along the Pt-Pt axis of the complex are not the direct recipients of excess energy, but channels of IVR to ligand deformation modes that can efficiently funnel it to the solvent were found to prevail. Thanks to the support of the vacuum $\triangle \mathrm{SCF} B O M D$ simulations and an analysis in terms of the time dependence of the internal vibrational kinetic energy along generalized normal modes, we were able to identify the intramolecular motions that are most likely to accept excess energy from the Pt-Pt pinching. The modes involved in the IVR were found to have main character of ligand twisting and Pt-P bond stretching, and vibrational periods between $\sim 120$ and $\sim 250 \mathrm{fs}$, close to the period of the Pt-Pt stretching vibrations ( $\sim 230$ fs from the simulations). The role of the solvent in the relaxation process was found to strengthen anharmonic couplings between the pinching and the ligand deformation modes, thus facilitating IVR with respect to the scenario in vacuum. The decay of coherent Pt-Pt oscillations in water from the simulations is around three times faster than the experimentally determined decoherence time; however, there is a qualitative agreement in the prediction that vibrational cooling along the Pt-Pt coordinate occurs by preserving to a large extent the vibrational phase.

The present study focused on the relaxation events taking place in $\mathrm{S}_{1}$ in the first picoseconds after photoexcitation in water. The intersystem crossing to the lower lying $\mathrm{T}_{1}$ state is known from transient absorption measurements to occur much later, after around $\sim 14$ ps. ${ }^{14}$ This permitted us to use $\triangle \mathrm{SCF}-\mathrm{QM} / \mathrm{MM}$ BOMD simulations that neglect any non-adiabatic and spin-orbit couplings between electronic states. The $\mathrm{S}_{1}$ state, where the investigated structural dynamics occurs, was found to be relatively well isolated from $T_{1}$ and other higher lying electronic states, as implied by the unperturbed shape of all obtained $\mathrm{S}_{1}$ potential curves. However, fluctuations in the solvent configurations could temporarily shift the energy levels, thus favouring the approach of different electronic states. In order to asses the interplay between these transient energy levels fluctuations and the structural 
distortion of the symmetry of the molecule caused by bending of the ligands in determining the rates of ISC in water solution, non-adiabatic dynamics simulations including SOCs and solvent effects are needed. Future computational studies should point in this direction to expand on the knowledge about the excited-state relaxation cascade at later times than those considered in the present investigation.

Concluding, we have shown that $\triangle \mathrm{SCF}-\mathrm{QM} / \mathrm{MM}$ BOMD simulations can be a very powerful tool to investigate aspects of the excited-state dynamics and reactivity of complex molecular systems in solution. Current computational resources pose a limit to the utilization of high-level multireference methods for statistically robust sampling of solvent-influenced dynamics. In this context, cost-effective multiscale methods based on DFT as presented here represent a valuable alternative, offering first-hand theoretical support to ultrafast experiments with unprecedented atomistic and time resolution.

\section{Acknowledgement}

The research leading to the presented results has received funding from the People Programme (Marie Curie Actions) of the European Union's Seventh Framework Programme (FP7/2007-2013) under REA grant agreement no. 609405 (COFUNDPostdocDTU), the Icelandic Research Fund (Grant 174244-051), the Villum Foundation, and the Danish Council for Independent Research.

\section{Supporting Information Available}

GPAW convergence tests, representation of the ground-state HOMO and LUMO orbitals of PtPOP, analysis of the $\mathrm{S}_{1}-\mathrm{S}_{0}$ electronic density difference, analysis of the energetics of $\mathrm{S}_{1}$ from the simulations, additional PES calculations, further details on the SF approximation and on the vibrational analysis (PDF)

Animation of the pinching mode (MPG) 
Animation 1 of the twist 1 mode (MPG)

Animation 2 of the twist 1 mode (MPG)

Animation 1 of the twist 2 mode (MPG)

Animation 2 of the twist 2 mode (MPG)

Animation 1 of the twist 3 mode (MPG)

Animation 2 of the twist 3 mode (MPG)

Animation of the breathing mode (MPG)

Animation of the bending mode (MPG) 


\section{References}

(1) Bergamini, G.; Silvi, S. Applied Photochemistry; Lecture Notes in Chemistry; Springer International Publishing, 2016; Vol. 92.

(2) Balzani, V.; Campagna, S. Photochemistry and Photophysics of Coordination Compounds I; Topics in Current Chemistry; Springer-Verlag Berlin Heidelberg, 2007; Vol. 280.

(3) Andreiadis, E. S.; Chavarot-Kerlidou, M.; Fontecave, M.; Artero, V. Artificial Photosynthesis: From Molecular Catalysts for Light-driven Water Splitting to Photoelectrochemical Cells. Photochemistry and Photobiology 2011, 87, 946-964.

(4) Nazeeruddin, M. K.; Baranoff, E.; Grätzel, M. Dye-sensitized solar cells: A brief overview. Solar Energy 2011, 85, 1172-1178.

(5) Sundstrom, V. Biophotonics: Spectroscopy, Imaging, Sensing, and Manipulation; NATO Science for Peace and Security Series B: Physics and Biophysics; Springer Netherlands, 2011; pp 219-236.

(6) Chergui, M.; Collet, E. Photoinduced Structural Dynamics of Molecular Systems Mapped by Time-Resolved X-ray Methods. Chemical Reviews 2017, 117, 11025-11065.

(7) Gray, H. B.; Záliš, S.; Vlček, A. Electronic structures and photophysics of d8-d8 complexes. Coordination Chemistry Reviews 2017, 345, 297-317.

(8) Chergui, M. Ultrafast Photophysics of Transition Metal Complexes. Accounts of Chemical Research 2015, 48, 801-808.

(9) Lemke, H. T.; Kjær, K. S.; Hartsock, R.; Driel, T. B. V.; Chollet, M.; Glownia, J. M.; Song, S.; Zhu, D.; Pace, E.; Matar, S. F. et al. Coherent structural trapping through wave packet dispersion during photoinduced spin state switching. Nature communications 2017, 8, 15342 . 
(10) Monni, R.; Auböck, G.; Kinschel, D.; Aziz-Lange, K. M.; Gray, H. B.; Vlček, A.; Chergui, M. Conservation of vibrational coherence in ultrafast electronic relaxation: The case of diplatinum complexes in solution. Chemical Physics Letters 2017, 683, $112-120$.

(11) Biasin, E.; van Driel, T. B.; Kjær, K. S.; Dohn, A. O.; Christensen, M.; Harlang, T.; Chabera, P.; Liu, Y.; Uhlig, J.; Pápai, M. et al. Femtosecond X-Ray Scattering Study of Ultrafast Photoinduced Structural Dynamics in Solvated [Co(terpy)2]2+. Physical Review Letters 2016, 117, 013002.

(12) Driel, T. B.; Kjær, K. S.; Hartsock, R.; Dohn, A. O.; Harlang, T.; Chollet, M.; Christensen, M.; Wojciech, G.; Henriksen, N. E.; Kim, J. G. et al. Atomistic characterization of the active-site solvation dynamics of a photocatalyst. Nature Communications 2016, ᄁ, 13678.

(13) Hua, L.; Iwamura, M.; Takeuchi, S.; Tahara, T. The substituent effect on the MLCT excited state dynamics of $\mathrm{Cu}(\mathrm{I})$ complexes studied by femtosecond time-resolved absorption and observation of coherent nuclear wavepacket motion. Phys. Chem. Chem. Phys. 2015, 17, 2067-2077.

(14) van der Veen, R. M.; Cannizzo, A.; van Mourik, F.; Vlcek, A. J.; Chergui, M. Vibrational relaxation and intersystem crossing of binuclear metal complexes in solution. Journal of the American Chemical Society 2011, 113, 305-315.

(15) Iwamura, M.; Watanabe, H.; Ishii, K.; Takeuchi, S.; Tahara, T. Coherent nuclear dynamics in ultrafast photoinduced structural change of Bis(diimine)copper(I) complex. Journal of the American Chemical Society 2011, 133, 7728-7736.

(16) Consani, C.; Prémont-Schwarz, M.; Elnahhas, A.; Bressler, C.; Van Mourik, F.; Cannizzo, A.; Chergui, M. Vibrational coherences and relaxation in the high-spin state 
of aqueous [FeII(bpy)3]2+. Angewandte Chemie - International Edition 2009, 48, $7184-7187$.

(17) Fumanal, M.; Gindensperger, E.; Daniel, C. Ultrafast Excited-State Decays in $[\operatorname{Re}(\mathrm{CO}) 3(\mathrm{~N}, \mathrm{~N})(\mathrm{L})] \mathrm{n}+$ : Nonadiabatic Quantum Dynamics. Journal of Chemical Theory and Computation 2017, 13, 1293-1306.

(18) Pápai, M.; Penfold, T. J.; Møller, K. B. Effect of tert-Butyl Functionalization on the Photoexcited Decay of a Fe(II)-N-Heterocyclic Carbene Complex. Journal of Physical Chemistry C 2016, 120, 17234-17241.

(19) Harabuchi, Y.; Eng, J.; Gindensperger, E.; Taketsugu, T.; Maeda, S.; Daniel, C. Exploring the Mechanism of Ultrafast Intersystem Crossing in Rhenium(I) Carbonyl Bipyridine Halide Complexes: Key Vibrational Modes and Spin-Vibronic Quantum Dynamics. Journal of Chemical Theory and Computation 2016, 12, 2335-2345.

(20) Pápai, M.; Vankó, G.; Rozgonyi, T.; Penfold, T. J. High Efficiency Iron Photosensitiser Explained with Quantum Wavepacket Dynamics. The Journal of Physical Chemistry Letters 2016, 7, 2009-2014.

(21) Eng, J.; Gourlaouen, C.; Gindensperger, E.; Daniel, C. Spin-Vibronic Quantum Dynamics for Ultrafast Excited-State Processes. Accounts of Chemical Research 2015, 48, 809-817.

(22) Beck, M. H.; Jckle, A.; Worth, G. A.; Meyer, H.-D. The multiconfiguration timedependent Hartree (MCTDH) method: A highly efficient algorithm for propagating wavepackets. Physics Reports 2000, 324, 1.

(23) Meyer, H. D.; Manthe, U.; Cederbaum, L. S. The multi-configurational time-dependent Hartree approach. Chemical Physics Letters 1990, 165, 73-78. 
(24) Mai, S.; Gattuso, H.; Fumanal, M.; Muñoz-Losa, A.; Monari, A.; Daniel, C.; Gonzalez, L. Excited-States of a Rhenium Carbonyl Diimine Complex: Solvation Models, Spin-Orbit Coupling, and Vibrational Sampling Effects. Phys. Chem. Chem. Phys. 2017, 3, 21-25.

(25) Atkins, A. J.; González, L. Trajectory Surface-Hopping Dynamics Including Intersystem Crossing in $[\mathrm{Ru}(\mathrm{bpy}) 3] 2+$. Journal of Physical Chemistry Letters 2017, 8, 3840-3845.

(26) Capano, G.; Penfold, T. J.; Chergui, M.; Tavernelli, I. Photophysics of a copper phenanthroline elucidated by trajectory and wavepacket-based quantum dynamics: a synergetic approach. Phys. Chem. Chem. Phys. 2017, 19, 19590-19600.

(27) Dohn, A. O.; Henriksen, N. E.; Møller, K. B. Transient Changes in Molecular Geometries and How to Model Them; Springer International Publishing, 2014.

(28) Tavernelli, I. Nonadiabatic Molecular Dynamics Simulations: Synergies between Theory and Experiments. Accounts of Chemical Research 2015, 48, 792-800.

(29) Dohn, A. O.; Jónsson, E. O.; Kjær, K. S.; B. van Driel, T.; Nielsen, M. M.; Jacobsen, K. W.; Henriksen, N. E.; Møller, K. B. Direct Dynamics Studies of a Binuclear Metal Complex in Solution: The Interplay Between Vibrational Relaxation, Coherence, and Solvent Effects. Journal of Physical Chemistry Letters 2014, 5, 2414-2418.

(30) Tavernelli, I.; Curchod, B. F. E.; Rothlisberger, U. Nonadiabatic molecular dynamics with solvent effects: A LR-TDDFT QM/MM study of ruthenium (II) tris (bipyridine) in water. Chemical Physics 2011, 391, 101-109.

(31) Daku, L. M. L.; Hauser, A. Ab Initio Molecular Dynamics Study of an Aqueous Solution of $\left[\mathrm{Fe}(\mathrm{bpy})_{3}\right](\mathrm{Cl})_{2}$ in the Low-Spin and in the High-Spin States. Journal of Physical Chemistry Letters 2010, 1, 1830-1835. 
(32) Moret, M.-E.; Tavernelli, I.; Rothlisberger, U. Combined QM/MM and Classical Molecular Dynamics Study of $[\mathrm{Ru}(\mathrm{bpy}) 3] 2+$ in Water. Journal of Physical Chemistry B 2009, 113, 7737-7744.

(33) Dominik Marx, J. H. Ab Initio Molecular Dynamics: Basic Theory and Advanced Methods; Cambridge University Press, 2009.

(34) Dohn, A. O.; Jonsson, E. Ö.; Levi, G.; Mortensen, J. J.; Lopez-Acevedo, O.; Thygesen, K. S.; Jacobsen, K. W.; Ulstrup, J.; Henriksen, N. E.; Møller, K. B. et al. Grid-Based Projector Augmented Wave (GPAW) Implementation of Quantum Mechanics/Molecular Mechanics (QM/MM) Electrostatic Embedding and Application to a Solvated Diplatinum Complex. Journal of Chemical Theory and Computation 2017, 13, 6010-6022.

(35) Dohn, A. O.; Kjær, K. S.; Harlang, T. B.; Canton, S. E.; Nielsen, M. M.; Møller, K. B. Electron Transfer and Solvent-Mediated Electronic Localization in Molecular Photocatalysis. Inorganic Chemistry 2016, 55, 10637-10644.

(36) Larsen, A. H.; Mortensen, J. J.; Blomqvist, J.; Castelli, I. E.; Christensen, R.; Dułak, M.; Friis, J.; Groves, M. N.; Hammer, B.; Hargus, C. et al. The atomic simulation environmenta Python library for working with atoms. Journal of Physics: Condensed Matter 2017, 29, 273002.

(37) Bahn, S. R.; Jacobsen, K. W. An object-oriented scripting interface to a legacy electronic structure code. Computing in Science \& Engineering 2002, 4, 55.

(38) Enkovaara, J.; Rostgaard, C.; Mortensen, J. J.; Chen, J.; Dulak, M.; Ferrighi, L.; Gavnholt, J.; Glinsvad, C.; Haikola, V.; Hansen, H. A. et al. Electronic structure calculations with GPAW: a real-space implementation of the projector augmentedwave method. Journal of Physics: Condensed matter 2010, 22, 253202. 
(39) Mortensen, J.; Hansen, L.; Jacobsen, K. W. Real-space grid implementation of the projector augmented wave method. Physical Review B 2005, 71, 035109.

(40) Ziegler, T.; Rauk, A.; Baerends, E. J. On the calculation of multiplet energies by the hartree-fock-slater method. Theoretica Chimica Acta 1977, 43, 261-271.

(41) Briggs, E. A.; Besley, N. A. Density Functional Theory Based Analysis of Photoinduced Electron Transfer in a Triazacryptand Based K+ Sensor. The Journal of Physical Chemistry A 2015, 2902-2907.

(42) Briggs, E. A.; Besley, N. A.; Robinson, D. QM/MM excited state molecular dynamics and fluorescence spectroscopy of BODIPY. Journal of Physical Chemistry A 2013, 117, 2644-2650.

(43) Kowalczyk, T.; Yost, S. R.; Voorhis, T. V. Assessment of the $\Delta$ SCF density functional theory approach for electronic excitations in organic dyes. Journal of Chemical Physics 2011, 134,054128 .

(44) Maurer, R. J.; Reuter, K. Assessing computationally efficient isomerization dynamics: $\triangle \mathrm{SCF}$ density-functional theory study of azobenzene molecular switching. Journal of Chemical Physics 2011, 135, 224303.

(45) Himmetoglu, B.; Marchenko, A.; Dabo, I.; Cococcioni, M. Role of electronic localization in the phosphorescence of iridium sensitizing dyes. Journal of Chemical Physics 2012, 137, 154309.

(46) Robinson, D.; Besley, N. A. Modelling the spectroscopy and dynamics of plastocyanin. Physical chemistry chemical physics 2010, 12, 9667-9676.

(47) Hanson-Heine, M. W. D.; George, M. W.; Besley, N. A. Calculating excited state properties using Kohn-Sham density functional theory. Journal of Chemical Physics 2013, 138 . 
(48) Gavnholt, J.; Olsen, T.; Engelund, M.; Schiøtz, J. Delta Self-Consistent Field as a Method to Obtain Potential Energy Surfaces of Excited Molecules on Surfaces. Physical Review B 2008, 78, 075441.

(49) Mendieta-Moreno, J. .; Trabada, D. G.; Mendieta, J.; Lewis, J. P.; Gómez-Puertas, P.; Ortega, J. Quantum Mechanics/Molecular Mechanics Free Energy Maps and Nonadiabatic Simulations for a Photochemical Reaction in DNA: Cyclobutane Thymine Dimer. Journal of Physical Chemistry Letters 2016, 7, 4391-4397.

(50) Stiegman, A. E.; Rice, S. F.; Gray, H. B.; Miskowski, V. M. Electronic spectroscopy of $\mathrm{d}^{8}-\mathrm{d}^{8}$ diplatinum complexes. ${ }^{1} \mathrm{~A}_{2 \mathrm{u}}\left(\mathrm{d} \sigma^{*} \rightarrow \mathrm{p} \sigma\right),{ }^{3} \mathrm{E}_{\mathrm{u}}\left(\mathrm{d}_{x z}, \mathrm{~d}_{y z} \rightarrow \mathrm{p} \sigma\right)$, and ${ }^{3,1} \mathrm{~B}_{2 \mathrm{u}}\left(\mathrm{d} \sigma^{*} \rightarrow\right.$ $\mathrm{d}_{x^{2}-y^{2}}$ excited states of $\mathrm{Pt}_{2}\left(\mathrm{P}_{2} \mathrm{O}_{5} \mathrm{H}_{2}\right)_{4}^{4-}$. Inorganic Chemistry 1987, 26, 1112.

(51) Rice, S. F.; Gray, H. B. Electronic Absorption and Emission Spectra of Binuclear Platinum(II) Complexes. Characterization of the Lowest Singlet and Triplet Excited States of $\mathrm{Pt}_{2}\left(\mathrm{P}_{2} \mathrm{O}_{5} \mathrm{H}_{2}\right)_{4}^{4-}$. J. Am. Chem. Soc 1983, 105, 4571-4575.

(52) Fordyce, W. A.; Brummer, J. G.; Crosby, G. A. Electronic Spectroscopy of a Diplatinum(II) Octaphosphite Complex. J. Am. Chem. Soc. 1981, 103, 7061-7064.

(53) Van Der Veen, R. M.; Milne, C. J.; Nahhas, A. E.; Lima, F. A.; Pham, V. T.; Best, J.; Weinstein, J. A.; Borca, C. N.; Abela, R.; Bressler, C. et al. Structural determination of a photochemically active diplatinum molecule by time-resolved EXAFS spectroscopy. Angewandte Chemie - International Edition 2009, 48, 2711-2714.

(54) Christensen, M.; Haldrup, K.; Bechgaard, K.; Feidenhans, R.; Kong, Q.; Cammarata, M.; Russo, M. L.; Wulff, M.; Harrit, N.; Nielsen, M. M. et al. Time-Resolved X-ray Scattering of an Electronically Excited State in Solution. Structure of the A State of Tetrakis- $\mu$-pyrophosphitodiplatinate (II). J. Am. Chem. Soc 2008, 131, 502508. 
(55) Yasuda, N.; Uekusa, H.; Ohashi, Y. X-ray analysis of excited-state structures of the diplatinum complex anions in five crystals with different cations. Bulletin of the Chemical Society of Japan 2004, 77, 933-944.

(56) Kim, C. D.; Pillet, S.; Wu, G.; Fullagar, W. K.; Coppens, P. Excited-state structure by time-resolved X-ray diffraction. Acta Crystallographica Section A: Foundations of Crystallography 2002, 58, 133-137.

(57) Ikeyama, T.; Yamamoto, S.; Azumi, T. Vibrational analysis of sublevel phosphorescence spectra of potassium tetrakis( $\mu$-diphosphonato)diplatinate(II): mechanism of radiative transition for the electronically forbidden A1u spectrum. The Journal of Physical Chemistry 1988, 92, 6899.

(58) Záliš, S.; Lam, Y.-C.; Gray, H. B.; Vlček, A. SpinOrbit TDDFT Electronic Structure of Diplatinum(II,II) Complexes. Inorganic Chemistry 2015, 54, 3491-3500.

(59) Novozhilova, I. V.; Volkov, A. V.; Coppens, P. Theoretical analysis of the triplet excited state of the [Pt2(H2P2O5)4]4- ion and comparison with time-resolved X-ray and spectroscopic results. Journal of the American Chemical Society 2003, 125, 10791087.

(60) Durrell, A. C.; Keller, G. E.; Lam, Y. C.; Sýkora, J.; Vlček, A.; Gray, H. B. Structural control of $1 \mathrm{~A} 2 \mathrm{u}$-to-3A2 $\mathrm{u}$ intersystem crossing in diplatinum(II,II) complexes. Journal of the American Chemical Society 2012, 134, 14201-14207.

(61) Milder, S. J.; Brunschwig, B. S. Factors Affecting Nonradiative Decay: Temperature Dependence of the Picosecond Fluorescence Lifetime of Pt2(pop)44-. The Journal of Physical Chemistry 1992, 96, 2189-2196.

(62) Roundhill, D. M.; Gray, H. B.; Che, C.-M. Pyrophosphito-Bridged Diplatinum Chemistry. Acc. Chem. Res 1989, 22, 55-61. 
(63) Strachan, A. Normal modes and frequencies from covariances in molecular dynamics or monte carlo simulations. Journal of Chemical Physics 2004, 120, 1.

(64) Maurer, R. J.; Reuter, K. Excited-state potential-energy surfaces of metal-adsorbed organic molecules from linear expansion $\Delta$-self-consistent field density-functional theory ( $\Delta$-DFT). Journal of Chemical Physics 2013, 139, 014708-014718.

(65) Rega, N. Vibrational analysis beyond the harmonic regime from Ab-initio molecular dynamics. Theoretical Chemistry Accounts 2006, 116, 347-354.

(66) Carbonniere, P.; Pouchan, C.; Improta, R. Intramolecular vibrational redistribution in the non-radiative excited state decay of uracil in the gas phase: an ab initio molecular dynamics study. Phys. Chem. Chem. Phys. 2015, 17, 11615-11626.

(67) Rega, N.; Brancato, G.; Petrone, A.; Caruso, P.; Barone, V. Vibrational analysis of x-ray absorption fine structure thermal factors by ab initio molecular dynamics: the $\mathrm{Zn}(\mathrm{II})$ ion in aqueous solution as a case study. The Journal of chemical physics 2011, 134,074504 .

(68) Kabsch, W. A solution for the best rotation to relate two sets of vectors. Acta Crystallographica Section A 1958, 32, 922-923.

(69) Dohn, A. O.; Møller, K. B.; Sauer, S. P. A. Optimizing the Structure of Tetracyanoplatinate (II): A Comparison of Relativistic Density Functional Theory Methods. Current Inorganic Chemistry 2013, 3, 213-219.

(70) Larsen, A. H.; Vanin, M.; Mortensen, J. J.; Thygesen, K. S.; Jacobsen, K. W. Localized atomic basis set in the projector augmented wave method. Phys. Rev. B 2009, 80, 195112.

(71) Gross, E. K. U.; Oliveira, L. N.; Kohn, W. Density-functional theory for ensembles of 
fractionally occupied states. I. Basic formalism. Physical Review A 1988, 37, 28092820.

(72) Becke, A. D. Density-Functional Exchange-Energy Approximation with Correct Asymptotic Behavior. Physical Review A 1988, 38, 3098.

(73) Lee, C.; Yang, W.; Parr, R. G. Development of the Colle-Salvetti correlation-energy formula into a functional of the electron density. Physical Review B 1988, 37, 785-789.

(74) Gellene, G. I.; Roundhill, D. M. Computational Studies on the Isomeric Structures in the Pyrophosphito Bridged Diplatinum (II) Complex , Platinum Pop. Journal of Physical Chemistry A 2002, 106, 7617-7620.

(75) G09 - Gaussian 09, Revision D.01, Frisch, M. J.; Trucks, G. W.; Schlegel, H. B.; Scuseria, G. E.; Robb, M. A.; Cheeseman, J. R.; Scalmani, G.; Barone, V.; Petersson, G. A.; Nakatsuji, H., et al. Gaussian, Inc., Wallingford CT, 2016.

(76) Schäfer, A.; Horn, R.; Ahlrichs, R. Fully optimized contracted gaussian basis sets for atoms Li to Kr. Journal of Chemical Physics 1992, 97, 2571-2577.

(77) Andrae, D.; Häußermann, U.; Dolg, M.; Stoll, H.; Preuß, H. T. Energy-adjusted ab initio pseudopotentials for the second and third row transition elements. Theor. Chim. Acta 1990, 77, 123-141.

(78) Weigend, F.; Ahlrichs, R. Balanced basis sets of split valence, triple zeta valence and quadruple zeta valence quality for H to Rn: Design and assessment of accuracy. Physical Chemistry, Chemical Physics 2005, 7, 3297-3305.

(79) Becke, A. D. Density-Functional Thermochemistry. III. The Role of Exact Exchange. J. Chem. Phys. 1993, 98, 5648-5652.

(80) Stephens, P. J.; Devlin, F. J.; Chabalowski, C. F.; Frisch, M. J. Ab-Initio Calculation 
of Vibrational Absorption and Circular-Dichroism Spectra Using Density-Functional Force-Fields. Journal of Physical Chemistry 1994, 98, 11623-11627.

(81) Andersen, H. C. Rattle: A "velocity" version of the shake algorithm for molecular dynamics calculations. Journal of Computational Physics 1983, 52, 24.

(82) Rappe, A.; Casewit, C.; Colwell, K.; III, W. G.; Skiff, W. UFF, a Full Periodic Table Force Field for Molecular Mechanics and Molecular Dynamics Simulations. Journal of the American Chemical Society 1992, 114, 10024-10035.

(83) Vanden-Eijnden, E.; Ciccotti, G. Second-order integrators for Langevin equations with holonomic constraints. Chemical Physics Letters 2006, 429, 310-316.

(84) Rey, R.; Hynes, J. T. Solvation Dynamics in Liquid Water. III. Energy Fluxes and Structural Changes. The journal of physical chemistry. B 2017, 121, 1377-1385.

(85) Rey, R.; Hynes, J. T. Solvation Dynamics in Water: 2. Energy Fluxes on Excited- and Ground-State Surfaces. The Journal of Physical Chemistry B 2016, 120, 11287-11297.

(86) Rey, R.; Hynes, J. T. Solvation Dynamics in Liquid Water. 1. Ultrafast Energy Fluxes. The Journal of Physical Chemistry B 2015, 119, 7558-7570.

(87) Petersen, J.; Henriksen, N. E.; Møller, K. B. Validity of the Bersohn-Zewail model beyond justification. Chemical Physics Letters 2012, 539-540, 234-238.

(88) Møller, K. B.; Rey, R.; Hynes, J. T. Hydrogen bond dynamics in water and ultrafast infrared spectroscopy: A theoretical study. Journal of Physical Chemistry A 2004, $108,1275-1289$.

(89) Ermoshin, V. A.; Engel, V. Femtosecond pump-probe fluorescence signals from classical trajectories: Comparison with wave-packet calculations. European Physical Journal D 2001, 15, 413-422. 
(90) Li, Z.; Fang, J.-Y.; Martens, C. C. Simulation of ultrafast dynamics and pump-probe spectroscopy using classical trajectories. The Journal of Chemical Physics 1996, 104, 6919.

(91) Jonas, D. M.; Bradforth, S. E.; Passino, S. A.; Fleming, G. R. Femtosecond Wavepacket Spectroscopy: Influence of Temperature, Wavelength, and Pulse Duration. Journal of Physical Chemistry 1995, 99, 2594-2608.

(92) Ozawa, Y.; Terashima, M.; Mitsumi, M.; Toriumi, K.; Yasuda, N.; Uekusa, H.; Ohashi, Y. Photoexcited Crystallography of Diplatinum Complex by Multipleexposure IP Method. Chemistry Letters 2003, 32, 62-63.

(93) Che, C. M.; Herbstein, F. H.; Schaefer, W. P.; Marsh, R. E.; Gray, H. B. Binuclear platinum diphosphite complexes. Crystal structures of $\mathrm{K} 4[\mathrm{Pt} 2(\mathrm{pop}) 4 \mathrm{Br}] \cdot 3 \mathrm{H} 2 \mathrm{O}$, a new linear chain semiconductor, and $\mathrm{K} 4[\mathrm{Pt} 2(\mathrm{pop}) 4 \mathrm{Cl} 2] \cdot 2 \mathrm{H} 2 \mathrm{O}$. Journal of the American Chemical Society 1983, 105, 4604-4607.

(94) Sadler, J.; Sanderson, M. R. A Novel Di-platinum(r1) Octaphosphite Complex showing Metal-Metal Bonding and Intense Luminescence; a Potential Probe for Basic Proteins. X-Ray Crystal and Molecular Structure. Journal of the Chemical Society, Chemical Communications 1980, 13-15.

(95) Berry, R. S. Correlation of Rates of Intramolecular Tunneling Processes, with Application to Some Group V Compounds. The Journal of Chemical Physics 1960, 32, 933-938.

(96) Alain, D.; Albright, T. A.; Hoffmann, R. Some Hydrido-Bridged Transition-Metal Dimers and Their Unsupported Analogues. Speculations on Pentuple Bonding and Pentuple Bridging. Journal of the American Chemical Society 1979, 101, 3141-3151.

(97) Van Der Veen, R. M.; Milne, C. J.; Pham, V.-T.; Nahhas, A. E.; Weinstein, J. A.; Best, J.; Borca, C. N.; Bressler, C.; Chergui, M. EXAFS Structural Determination of 
the Pt2(P2O5H2)44- Anion in Solution. CHIMIA International Journal for Chemistry 2008, 62, 287-290.

(98) Biasin, E.; Driel, T. B. V.; Levi, G.; Laursen, M. G.; Dohn, A. O.; Moltke, A.; Vester, P.; Hansen, F. B. K.; Kjaer, K. S.; Hartsock, R. et al. Anisotropy enhanced X-ray scattering from solvated transition metal complexes. Journal of Synchrotron Radiation 2017, 25, 2.

(99) Peterson, J. R.; Kalyanasundaram, K. Energy- and Electron-Transfer Processes of the Lowest Triplet Excited State of Tetrakis(diphosphito)diplatinate(II). Journal of Physical Chemistry 1985, 89, 2486-2492.

(100) Stein, P.; Dickson, M. K.; Roundhill, D. M. Raman and Infrared Spectra of Binuclear Platinum(II) and Platinum(III) Octaphosphite Complexes. A Characterization of the Intermetallic Bonding. J. Am. Chem. Soc. 1983, 105, 3489-3494.

(101) Kohen, D.; Tannor, D. J. Classical-quantum correspondence in the Redfield equation and its solutions. Journal of Chemical Physics 1997, 107, 5141-5153.

(102) Jean, J. M.; Fleming, G. R. Competition between energy and phase relaxation in electronic curve crossing processes. Journal of Chemical Physics 2013, 103, 20922101.

(103) Lee, J. H.; Wulff, M.; Bratos, S.; Petersen, J.; Guerin, L.; Leicknam, J. C.; Cammarata, M.; Kong, Q.; Kim, J.; Møller, K. B. et al. Filming the birth of molecules and accompanying solvent rearrangement. Journal of the American Chemical Society 2013, 135, 3255-3261.

(104) Zadoyan, R.; Li, Z.; Martens, C. C.; Apkarian, V. A. The breaking and remaking of a bond: Caging of I2 in solid Kr. Journal of Chemical Physics 1994, 101, 6648-6657. 


\section{TOC Graphic}

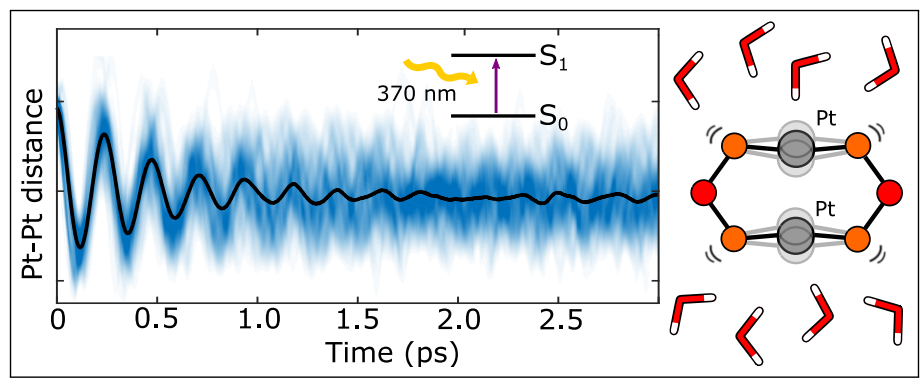

